\title{
The energetics response to a warmer climate: relative contributions from the transient and stationary eddies
}

\author{
D. Hernández-Deckers ${ }^{1,2}$ and J.-S. von Storch ${ }^{1}$ \\ ${ }^{1}$ Max Planck Institute for Meteorology, Hamburg, Germany \\ ${ }^{2}$ International Max Planck Research School on Earth System Modelling, Hamburg, Germany \\ Received: 1 April 2011 - Published in Earth Syst. Dynam. Discuss.: 8 April 2011 \\ Revised: 15 June 2011 - Accepted: 15 June 2011 - Published: 21 June 2011
}

\begin{abstract}
We use the Lorenz Energy Cycle (LEC) to evaluate changes in global energetic activity due to $\mathrm{CO}_{2}$ doubling in the coupled atmosphere-ocean ECHAM5/MPIOM model. Globally, the energetic activity - measured as the total conversion rate of available potential energy into kinetic energy - decreases by about $4 \%$. This weakening results from a dual response that consists of a strengthening of the LEC in the upper-troposphere and a weakening in the lower and middle troposphere. This is fully consistent with results from a coarser resolution version of the same coupled model. We further use our experiments to investigate the individual contributions of the transient and stationary eddy components to the main energetics response.

The transient eddy terms have a larger contribution to the total energetic activity than the stationary ones. We find that this is also true in terms of their $2 \times \mathrm{CO}_{2}$-response. Changes in the transient eddy components determine the main energetics response, whereas the stationary eddy components have very small contributions. Hence, the dual response strengthening in the upper troposphere and weakening below - concerns mainly the transient eddy terms. We can relate qualitatively this response to the two main features of the $2 \times \mathrm{CO}_{2}$ warming pattern: (a) the tropical uppertropospheric warming increases the pole-to-equator temperature gradient - strengthening the energetic activity above and enhances static stability - weakening the energetic activity below; and (b) the high-latitude surface warming decreases the pole-to-equator temperature gradient in the lower troposphere - weakening the energetic activity below. Despite the small contribution from the stationary eddies to the main energetics response, changes in stationary eddy
\end{abstract}

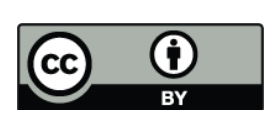

Correspondence to:

D. Hernández-Deckers

(daniel.hernandez@zmaw.de) available potential energy $\left(P_{\mathrm{se}}\right)$ reflect some features of the warming pattern: stronger land-sea contrasts at the subtropics and weaker land-sea contrasts at the high northern latitudes affect $P_{\text {se }}$ regionally, but do not affect the global energetics response.

\section{Introduction}

In a previous study, Hernández-Deckers and von Storch (2010) (hereafter referred to as HDvS) analyse the atmospheric energetics response to higher greenhouse gas concentrations using a coarse resolution version of the coupled atmosphere-ocean ECHAM5/MPI-OM model (T31L19 resolution for the atmospheric component and GR30L40 for the ocean component). By computing changes in the Lorenz Energy Cycle (LEC) (Lorenz, 1955), they find an overall weakening in energetic activity, measured as a global decrease in the total conversion of available potential energy $(P)$ into kinetic energy $(K)$. This result agrees with other studies (Boer, 1995; Marquet, 2006; Lucarini et al., 2010), which attribute this reduced energetic activity to a more isothermal atmosphere. That is, higher $\mathrm{CO}_{2}$ concentrations result in a reduced pole-to-equator temperature gradient and in smaller land-sea contrasts during the winter season. Such effects are expected to reduce baroclinic activity in a global scale. Nevertheless, HDvS find that the global weakening of the energetic activity consists of a strengthening in the upper troposphere and a weakening in the lower and middle troposphere, the latter dominating the globally-integrated picture. Their analysis indicates that this dual response is closely related to specific features of the warming pattern. In particular, not only the high-latitude surface warming contributes to the weakening of the energetic activity, but also the

Published by Copernicus Publications on behalf of the European Geosciences Union. 
increased mean static stability caused by the tropical uppertropospheric warming contributes to the overall weakening.

The LEC, formulated by Lorenz (1955), is the classical formalism to estimate the working of the atmosphere as a heat engine. Based on the concept of available potential energy $(P)$, it describes how the general circulation of the atmosphere is maintained against frictional dissipation from a global point of view. The LEC consists of three main steps: the generation of $P$ through differential heating, its conversion into kinetic energy $(K)$ by rising of relatively warm air and sinking of relatively cold air, and its dissipation by friction. One can further decompose the $P$ and $K$ reservoirs into zonal-mean and eddy components $\left(P_{\mathrm{m}}, P_{\mathrm{e}}, K_{\mathrm{m}}\right.$, and $\left.K_{\mathrm{e}}\right)$, obtaining a better insight into the processes which are important in terms of the energetics. In particular, baroclinic instability is the main process responsible for the total conversion of $P$ into $K$ through the path $P_{\mathrm{m}} \rightarrow P_{\mathrm{e}} \rightarrow K_{\mathrm{e}}$. That is, the largest conversion rate of $P$ into $K$ is $C\left(P_{\mathrm{e}}, K_{\mathrm{e}}\right)$, the conversion rate between $P_{\mathrm{e}}$ and $K_{\mathrm{e}}$ (Lorenz, 1955; Peixoto and Oort, 1974). The conversion rate between $P_{\mathrm{m}}$ and $K_{\mathrm{m}}, C\left(P_{\mathrm{m}}, K_{\mathrm{m}}\right)$, is related to meridional overturning circulations: $P_{\mathrm{m}}$ is converted into $K_{\mathrm{m}}$ in the thermally direct Hadley cell, whereas the opposite conversion takes place in the thermally indirect Ferrel cells (Li et al., 2007). When globally-integrated, $C\left(P_{\mathrm{m}}, K_{\mathrm{m}}\right)$ is small compared to $C\left(P_{\mathrm{e}}, K_{\mathrm{e}}\right)$. Therefore, we focus here on the energetics along the path $P_{\mathrm{m}} \rightarrow P_{\mathrm{e}} \rightarrow K_{\mathrm{e}} \rightarrow K_{\mathrm{m}}$. Furthermore, both observations and model studies point out that most of the energetic activity along this path concerns the transient eddy components, whereas the stationary eddy components contribute with a much smaller fraction of the global energy conversions (Oort and Peixoto, 1974; Holton, 2004). However, the energetic response to higher $\mathrm{CO}_{2}$ concentrations is not necessarily dominated by the transient eddy component's response. In principle, transient and stationary eddy components may be affected in a different way by a warmer climate. For example, the stronger warming over the continents could affect the stationary wave activity. It is not clear whether this is the case, and if yes, to what extent these changes contribute to the global energetics response. Therefore, an important step in order to fully understand the energetics response to higher $\mathrm{CO}_{2}$ concentrations is to distinguish between the response of the transient and the stationary eddy components. In order to investigate this within the framework of the LEC, here we carry out a decomposition of the eddy reservoirs that was not done by HDvS. We decompose them into transient and stationary eddy available potential energy $\left(P_{\mathrm{te}}\right.$ and $\left.P_{\mathrm{se}}\right)$, and into transient and stationary eddy kinetic energy $\left(K_{\text {te }}\right.$ and $\left.K_{\text {se }}\right)$, and we evaluate how this decomposed-LEC responds to a doubling of $\mathrm{CO}_{2}$ concentrations. In order to obtain a better representation of the eddies, we use here a higher resolution version of the coupled atmosphere-ocean ECHAM5/MPI-OM model. Therefore, we will first briefly verify HDvS's results with our higher resolution version of the model, and then perform the additional transient and stationary eddy decomposition to determine the role each of them plays in the main energetics response.

The following section describes the method we use, in particular the way in which we treat the LEC. Section 3 presents the results: we first analyse the main $2 \times \mathrm{CO}_{2}$ energetics response, verifying the results obtained by $\mathrm{HDvS}$ with a coarser resolution version of the coupled model. Second, we present the results concerning the new transient and stationary eddy decomposition of the LEC. Finally, Sect. 4 corresponds to the Conclusions and discussion. We include an Appendix with the description of the equations we use to compute the different LEC terms.

\section{Method}

\subsection{Model and experiments}

We analyse experiments carried out with the coupled atmosphere-ocean ECHAM5/MPI-OM general circulation model developed at the Max Planck Institute for Meteorology in Hamburg. The atmospheric component ECHAM5.2.02a (Roeckner et al., 2003) - has a T63L31 spectral resolution $\left(\approx 1.875^{\circ} \times 1.875^{\circ}\right)$ and 31 vertical levels. The ocean component - MPI-OM version 1.0 (Marsland et al., 2003) - has a GR15L40 resolution $\left(\approx 1.5^{\circ} \times 1.5^{\circ}\right)$ and 40 vertical levels. We use two integrations performed for the IPCC fourth assessment report:

- The last 50 years (from a total of 505 years) of the pre-industrial control experiment PIcntrl (Roeckner et al., 2006), with a constant $1 \times \mathrm{CO}_{2}$ concentration of $280 \mathrm{ppm}$.

- The last 50 years of the $1 \% \mathrm{yr}^{-1} \mathrm{CO}_{2}$-increase experiment to doubling (run no. 1) (Roeckner, 2004) $\left(\mathrm{CO}_{2^{-}}\right.$ doubling is achieved after 70 years and kept constant for 150 additional years).

We refer to these two experiments as " $1 \times \mathrm{CO}_{2}$ control run" and " $2 \times \mathrm{CO}_{2}$ experiment", respectively.

\subsection{Lorenz Energy Cycle equations}

The Lorenz Energy Cycle (LEC) equations are fundamentally the same as those considered by HDvS, but include the transient and stationary eddy decomposition:

$$
\begin{aligned}
\frac{\mathrm{d} P_{\mathrm{m}}}{\mathrm{d} t} & =-C\left(P_{\mathrm{m}}, P_{\mathrm{se}}\right)-C\left(P_{\mathrm{m}}, P_{\mathrm{te}}\right)-C\left(P_{\mathrm{m}}, K_{\mathrm{m}}\right) \\
& +G_{\mathrm{m}}+B\left(P_{\mathrm{m}}\right) \\
\frac{\mathrm{d} P_{\mathrm{se}}}{\mathrm{d} t} & =C\left(P_{\mathrm{m}}, P_{\mathrm{se}}\right)+C\left(P_{\mathrm{te}}, P_{\mathrm{se}}\right)-C\left(P_{\mathrm{se}}, K_{\mathrm{se}}\right) \\
& +G_{\mathrm{se}}+B\left(P_{\mathrm{se}}\right)
\end{aligned}
$$




$$
\begin{aligned}
\frac{\mathrm{d} P_{\mathrm{te}}}{\mathrm{d} t} & =C\left(P_{\mathrm{m}}, P_{\mathrm{te}}\right)-C\left(P_{\mathrm{te}}, P_{\mathrm{se}}\right)-C\left(P_{\mathrm{te}}, K_{\mathrm{te}}\right) \\
& +G_{\mathrm{te}}+B\left(P_{\mathrm{te}}\right) \\
\frac{\mathrm{d} K_{\mathrm{se}}}{\mathrm{d} t} & =C\left(P_{\mathrm{se}}, K_{\mathrm{se}}\right)-C\left(K_{\mathrm{se}}, K_{\mathrm{te}}\right)-C\left(K_{\mathrm{se}}, K_{\mathrm{m}}\right) \\
& -D_{\mathrm{se}}+B\left(K_{\mathrm{se}}\right) \\
\frac{\mathrm{d} K_{\mathrm{te}}}{\mathrm{d} t} & =C\left(P_{\mathrm{te}}, K_{\mathrm{te}}\right)+C\left(K_{\mathrm{se}}, K t e\right)-C\left(K_{\mathrm{te}}, K_{\mathrm{m}}\right) \\
& -D_{\mathrm{te}}+B\left(K_{\mathrm{te}}\right) \\
\frac{\mathrm{d} K_{\mathrm{m}}}{\mathrm{d} t} & =C\left(K_{\mathrm{se}}, K_{\mathrm{m}}\right)+C\left(K_{\mathrm{te}}, K_{\mathrm{m}}\right)+C\left(P_{\mathrm{m}}, K_{\mathrm{m}}\right) \\
& -D_{\mathrm{m}}+B\left(K_{\mathrm{m}}\right) .
\end{aligned}
$$

Here $P$ stands for the reservoir of available potential energy and $K$ for the reservoir of kinetic energy, both measured in $\mathrm{J} \mathrm{m}^{-2}$. The subscript $m$ stands for zonal mean component, the subscript "se" for stationary eddy component, and the subscript "te" for transient eddy component. This means that the eddy reservoirs considered by HDvS are just the sum of the stationary and transient eddy reservoirs: $P_{\mathrm{e}}=P_{\mathrm{se}}+P_{\mathrm{te}}$ and $K_{\mathrm{e}}=K_{\mathrm{se}}+K_{\mathrm{te}}$. The terms of the form $C(X, Y)$ represent the conversion rate of the reservoir $X$ into the reservoir $Y$, measured in $\mathrm{W} \mathrm{m}^{-2}$. The terms of the form $G_{x}$ indicate the generation rate of the x-component of available potential energy measured in $\mathrm{W} \mathrm{m}^{-2}$. For example, $G_{\mathrm{se}}$ is the generation rate of $P_{\mathrm{se}}$, whereas $G_{\mathrm{m}}$ is the generation rate of $P_{\mathrm{m}}$. In the same way, the terms of the form $D_{x}$ indicate the dissipation rate of the $\mathrm{x}$-component of kinetic energy. The terms $B(X)$ stand for boundary fluxes of the corresponding reservoir $X$ for the case in which the domain is not the whole atmosphere, and hence a boundary is specified. For global integrals, these boundary flux terms vanish.

Compared to HDvS, we only consider two entirely new terms. They correspond to the conversion rates between the stationary and transient components of each eddy reservoir: $C\left(P_{\mathrm{te}}, P_{\mathrm{se}}\right)$ and $C\left(K_{\mathrm{se}}, K_{\mathrm{te}}\right)$. The other "new" terms are simply a decomposition of old terms (e.g., $\left.C\left(P_{\mathrm{m}}, P_{\mathrm{e}}\right)=C\left(P_{\mathrm{m}}, P_{\mathrm{se}}\right)+C\left(P_{\mathrm{m}}, P_{\mathrm{te}}\right)\right)$. The exact expressions for the different terms are given in Appendix A. They are fully consistent with the expressions used by HDvS, as well as with the formulations of Peixoto and Oort (1974) and of Boer and Lambert (2008).

\subsection{Decompositions}

Our formulation is based on the Eulerian mean decomposition, where $X=\langle X\rangle+X^{\prime}$ and $\langle X\rangle=[\langle X\rangle]+\langle X\rangle^{*}$, so that $X=[\langle X\rangle]+\langle X\rangle^{*}+X^{\prime}$. Here $\langle X\rangle$ represents the time mean of the quantity $X, X^{\prime}$ denotes the deviation from this time mean, $[\langle X\rangle]$ denotes the zonal mean of $\langle X\rangle$, and $\langle X\rangle^{*}$, the deviation from this zonal mean. Additionally, we also need the decomposition into global mean over a constant pressure level (denoted by $\widetilde{X}$ ) and its deviation (denoted by $X^{\prime \prime}$ ), as these are required in Lorenz's approximation equation for available potential energy (see Appendix A). One has $[\langle X\rangle]^{\prime \prime}=[\langle X\rangle]-\langle\widetilde{X}\rangle$.

Note that the stationary and transient eddy decomposition distinguishes quadratic terms of the form $\left[\langle X\rangle^{* 2}\right]$ for the stationary eddy terms from terms of the form $\left[X^{\prime 2}\right]$ for the transient eddy terms. The stationary eddy components describe departures from the zonal-mean field that are persistent in time, and the transient eddy components describe the zonal mean of departures from the time-mean field. In the atmosphere, stationary eddies appear due to spatial inhomogeneities like topography and the position of continents; transient eddies result from dynamical instabilities and are related to storm activity.

\subsection{LEC computations}

The LEC-terms we compute here are the reservoirs $P_{\mathrm{m}}, P_{\mathrm{se}}$, $P_{\mathrm{te}}, K_{\mathrm{se}}, K_{\mathrm{te}}$, and $K_{\mathrm{m}}$, the conversion rates $C\left(P_{\mathrm{m}}, P_{\mathrm{se}}\right)$, $C\left(P_{\mathrm{m}}, P_{\mathrm{te}}\right), C\left(P_{\mathrm{te}}, P_{\mathrm{se}}\right), \quad C\left(P_{\mathrm{se}}, K_{\mathrm{se}}\right), C\left(P_{\mathrm{te}}, K_{\mathrm{te}}\right)$, $C\left(K_{\mathrm{se}}, K_{\mathrm{te}}\right), C\left(K_{\mathrm{se}}, K_{\mathrm{m}}\right), C\left(K_{\mathrm{te}}, K_{\mathrm{m}}\right)$ and $C\left(P_{\mathrm{m}}, K_{\mathrm{m}}\right)$, and, when splitting the atmosphere into upper and lower regions, the boundary flux terms $B\left(P_{\mathrm{m}}\right), B\left(P_{\mathrm{e}}\right), B\left(K_{\mathrm{e}}\right)$, and $B\left(K_{\mathrm{m}}\right)$. The generation and dissipation rates $G_{\mathrm{m}}, G_{\mathrm{se}}, G_{\mathrm{te}}$, $D_{\mathrm{se}}, D_{\mathrm{te}}$, and $D_{\mathrm{m}}$ are estimated as residuals assuming constant reservoirs. In other words, the left hand side of Eqs. (1) through (6) is equal to zero assuming equilibrium conditions, so that the generation and dissipation terms can be estimated as residuals of the remaining terms.

In the first part of this paper we consider the transient and stationary eddy terms together in one eddy term. For example, we consider $P_{\mathrm{e}}$ instead of $P_{\mathrm{te}}$ and $P_{\mathrm{se}}$, and the conversion terms $C\left(P_{\mathrm{m}}, P_{\mathrm{e}}\right)$, instead of $C\left(P_{\mathrm{m}}, P_{\mathrm{se}}\right)$ and $C\left(P_{\mathrm{m}}, P_{\mathrm{te}}\right)$. This ensures a consistent comparison with the results from HDvS. In all cases, we use 50 years of daily data from each run to obtain one value for each LEC-term. Therefore, the values presented here reflect the mean energetics of a 50year period, and the eddies we refer to are obtained as the deviations of daily values from a 50-year mean. Hence, their time-scales may range from a few days to several years.

\section{Results}

\subsection{The main $2 \times \mathrm{CO}_{2}$ energetics response}

Before dealing with the transient and stationary eddy decomposition of the Lorenz Energy Cycle (LEC), we briefly describe here the main energetics response in terms of the usual 2 and 4-box LEC diagrams. This serves as a verification of HDvS's results with a different resolution of the same coupled model, and at the same time it provides a more adequate setup - due to the higher resolution - to analyse the eddy contributions in the next subsections. 

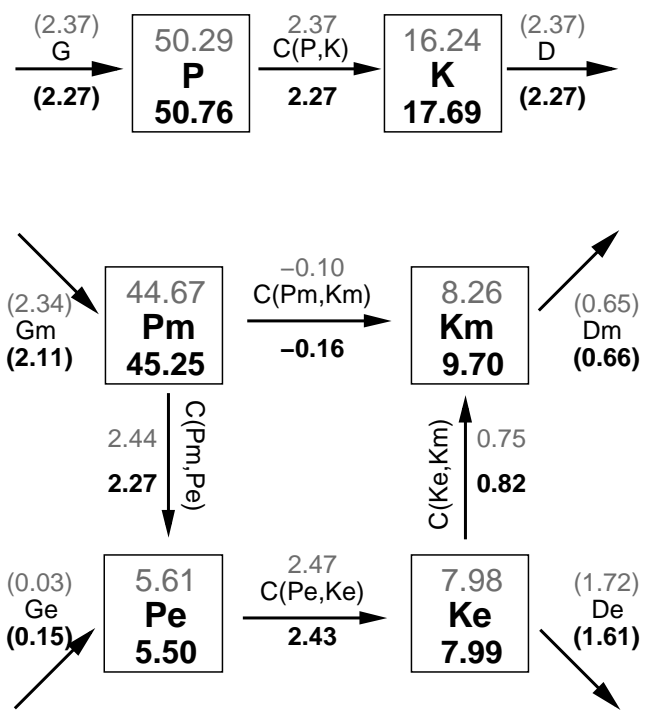
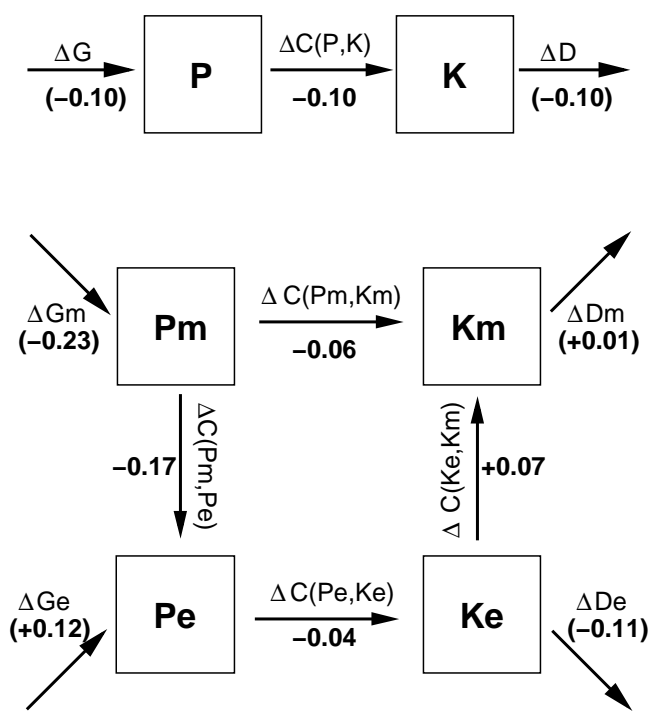

Fig. 1. 2-box (above) and 4-box (below) diagrams of the LEC. Left panel shows values for the $1 \times \mathrm{CO}_{2}$ control run (above, gray) and the $2 \times \mathrm{CO}_{2}$ equilibrium run (below, black). Generation and dissipation terms (in parenthesis) are obtained as residuals. Right panel shows the changes in energy generation, conversion and dissipation rates when doubling $\mathrm{CO}_{2}$ concentrations. Units are $10^{5} \mathrm{~J} \mathrm{~m}^{-2}$ for reservoirs and $\mathrm{W} \mathrm{m}^{-2}$ for conversion, generation and dissipation terms. Arrows indicate the direction corresponding to positive values; negative values imply opposite direction.

The values obtained for the LEC-terms in the $1 \times \mathrm{CO}_{2}$ and $2 \times \mathrm{CO}_{2}$ experiments (Fig. 1, left panel) differ slightly from the values obtained previously by HDvS. However, we do not expect exactly the same numbers in both cases because of the difference in resolution and model versions. For example, the total conversion rate $C(P, K)$ of our $1 \times \mathrm{CO}_{2}$ control experiment is $2.37 \mathrm{~W} \mathrm{~m}^{-2}$, compared to $2.66 \mathrm{~W} \mathrm{~m}^{-2}$ in the coarse resolution $1 \times \mathrm{CO}_{2}$ control run (HDvS). What we do expect is that the response of the LEC-terms to a $\mathrm{CO}_{2}$ doubling is similar. The changes in energy generation, conversion and dissipation terms (Fig. 1, right panel) constitute this energetics response due to $\mathrm{CO}_{2}$ doubling. These changes are indeed very similar to the ones obtained by HDvS. For example, the LEC-strength - the total $P$ to $K$ conversion rate $(C(P, K))$ - decreases by $4.2 \%$, compared to $6.8 \%$ obtained by HDvS. It indicates a global weakening of the energetic activity, although slightly less pronounced as with the coarse resolution model (HDvS). Regarding the 4-box diagram, the energetics response is also consistent in both cases. Just as HDvS, we find a weakening along the baroclinic path of the cycle $\left(P_{\mathrm{m}} \rightarrow P_{\mathrm{e}} \rightarrow K_{\mathrm{e}}\right)$, together with a strengthening of the barotropic-related conversion rate $C\left(K_{\mathrm{e}}, K_{\mathrm{m}}\right)$. The weakening response is slightly less pronounced here than in the coarser resolution results: $C\left(P_{\mathrm{m}}, P_{\mathrm{e}}\right)$ and $C\left(P_{\mathrm{e}}, K_{\mathrm{e}}\right)$ decrease here by $7 \%$ and $1.6 \%$ compared to $11 \%$ and $3.9 \%$ in HDvS. On the other hand, the strengthening response in $C\left(K_{\mathrm{e}}, K_{\mathrm{m}}\right)$ is more pronounced here than in the coarser resolution results; it strengthens by $9.3 \%$ here, compared to $5.1 \%$ in HDvS. The main energetics response is clearly consistent in both cases, and the small differences we find concern mainly the magnitude of the response.

HDvS conclude that the warming pattern strongly determines the energetics response. Therefore, this difference in magnitude can be easily explained with the differences in the warming pattern. In our experiments, the amplitude of the warming (Fig. 2) is smaller than in the coarse resolution experiments. On average, the warming we find is about 1$1.5 \mathrm{~K}$ lower than in the coarse resolution experiments analysed by HDvS, but the zonal-mean pattern, with the strongest warming in the upper tropical troposphere and in the surface high-latitude regions (mostly in the Northern Hemisphere) is the same. This could explain why we observe a slightly weaker but consistent response in comparison to HDvS. Several factors may be responsible for the difference in the amplitude of the $2 \times \mathrm{CO}_{2}$ warming. First of all, the experiments were carried out not only with different resolutions, but also with different versions of the model. Therefore, there may be slight differences in several parameterization schemes and in the tuning of the model. Furthermore, the period of time in which the $2 \times \mathrm{CO}_{2}$ concentration is held constant in both cases is not the same. Our $2 \times \mathrm{CO}_{2}$ run was obtained by increasing the $\mathrm{CO}_{2}$ concentration by $1 \%$ per year during 70 years, and then holding it constant for 150 years. We use here the last 50 years of this integration. In contrast to this, the coarse resolution $2 \times \mathrm{CO}_{2}$ experiment (HDvS) has 880 years of integration with constant $2 \times \mathrm{CO}_{2}$ concentration, of which they use the last 100 years. This longer equilibrium integration certainly accounts for some 


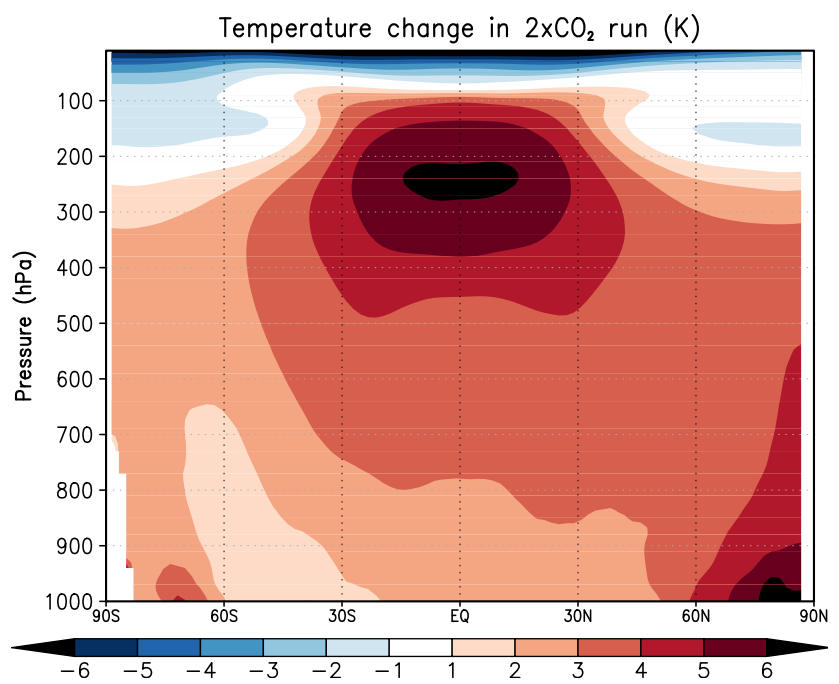

Fig. 2. Zonal-mean temperature change due to doubling of $\mathrm{CO}_{2}$ concentrations in the coupled atmosphere-ocean ECHAM5/MPIOM model.

further warming as the deep ocean approaches its equilibrium temperature. Nevertheless, the fact that the warming pattern in both cases is the same, suggests that the temperature response in the atmosphere in both cases is consistent, although with slightly different amplitudes.

HDvS found that this overall weakening of the energetic activity consists of a strengthening in the upper troposphere and a weakening below, the latter being the dominant globally-integrated response. The vertical cross-sections of the different LEC-terms (Fig. 3) suggest this same feature in our results. Clearly, this strengthening and weakening feature is present along the path $P_{\mathrm{m}} \rightarrow P_{\mathrm{e}} \rightarrow K_{\mathrm{e}}$, just as in the coarse resolution results (HDvS). The biggest difference we can detect is in the Southern Hemisphere $P_{\mathrm{m}}$-response. In the low resolution runs there is a general decrease, except for a small region between $60^{\circ} \mathrm{S}$ and $25^{\circ} \mathrm{S}$ near the surface, where $P_{\mathrm{m}}$ slightly increases. This region of $P_{\mathrm{m}}$-increase extends higher up in the higher resolution runs, with a maximum around $600 \mathrm{hPa}$. It resembles to some extent the transient response analysed by HDvS, where $P_{\mathrm{m}}$ increased throughout the whole troposphere in the Southern Hemisphere. In that case, this North-South asymmetry in the $P_{\mathrm{m}}$-change was attributed to a slower warming in the Southern Hemisphere (HDvS) due to a negative feedback in the Southern Ocean (von Storch, 2008). Although not as strong as in those transient experiments, this feature might be due to the fact that the equilibrium time of our $2 \times \mathrm{CO}_{2}$ experiment is shorter, and because the warming is weaker than in the low resolution runs. In any case, these vertical cross-sections suggest the same upper-tropospheric strengthening and lowertropospheric weakening response found by HDvS.

Furthermore, in order to verify this feature in terms of integrated LEC-terms, we split the atmosphere at $340 \mathrm{hPa}$ and compute the different LEC-terms in each region, now including the corresponding boundary flux terms (see Appendix). We use here $340 \mathrm{hPa}$ instead of $350 \mathrm{hPa}$ level (as HDvS use) because in our resolution this level corresponds to a modellevel, which facilitates the computations.

We have computed the LEC-terms for the upper and lower regions (not shown here) in order to obtain from these the corresponding changes in the generation, conversion, dissipation rates and boundary fluxes (Fig. 4). These changes describe the energetics response to a doubling of $\mathrm{CO}_{2}$ concentrations. Overall, we do observe a strengthening of the LEC terms in the upper region and a weakening in the lower region. Comparing our split-atmosphere's energetics response with the coarse resolution results (HDvS), we see a clear consistency with only one remark: the weakening response of the lower region is less pronounced here, whereas the strengthening response of the upper region has a similar magnitude in both cases. The total conversion rate $C(P, K)$ increases by $0.09 \mathrm{~W} \mathrm{~m}^{-2}$ above $340 \mathrm{hPa}$, and decreases by $0.19 \mathrm{~W} \mathrm{~m}^{-2}$ below. HDvS reported an increase of $0.10 \mathrm{~W} \mathrm{~m}^{-2}$ in the upper region and a decrease of $0.29 \mathrm{~W} \mathrm{~m}^{-2}$ below. This difference in the response of the lower and middle troposphere may be due to the differences in the amplitude of the warming pattern. Nevertheless, both overall responses are similar enough as to be considered consistent with each other: all the terms related to the baroclinicpath of the cycle, $P_{\mathrm{m}}, C\left(P_{\mathrm{m}}, P_{\mathrm{e}}\right), P_{\mathrm{e}}, C\left(P_{\mathrm{e}}, K_{\mathrm{e}}\right)$ and $K_{\mathrm{e}}$ show an increase in the upper region and a decrease below, whereas the $K_{\mathrm{e}}$-to- $K_{\mathrm{m}}$ conversion rate increases in the upper region and remains almost unchanged below. Clearly, the response of $G_{\mathrm{m}}$ is the strongest in both regions, suggesting that this term is driving the whole energetics response.

Summing up, evaluating the LEC for the split-atmosphere confirms what the vertical cross-sections (Fig. 3) suggest: the LEC strengthens in the upper-troposphere (roughly above $350 \mathrm{hPa}$ ), but weakens below. The weakening is visible in the baroclinic path of the cycle, while the strengthening is visible in both the baroclinic path and the barotropic $K_{\mathrm{e}}$-to- $K_{\mathrm{m}}$ conversion rate. Furthermore, both responses seem to be driven by changes in $G_{\mathrm{m}}$.

\subsection{Transient and stationary eddy decomposition}

Until here we have carried out the usual LEC analysis without decomposing the transient and stationary eddy components, and we find a general consistency with the results obtained by HDvS. We will now expand these conclusions by investigating the different contributions of the transient and stationary eddy components of the LEC. This should enable us to quantify the individual contribution of these components to the full response of the $\mathrm{LEC}$ to a $\mathrm{CO}_{2}$-doubling.

The values of the corresponding LEC terms in this case (Fig. 5) show that the eddy activity is dominated by the transient eddy terms. This is true regarding both reservoirs and conversion terms in both the $1 \times \mathrm{CO}_{2}$ and $2 \times \mathrm{CO}_{2}$ 

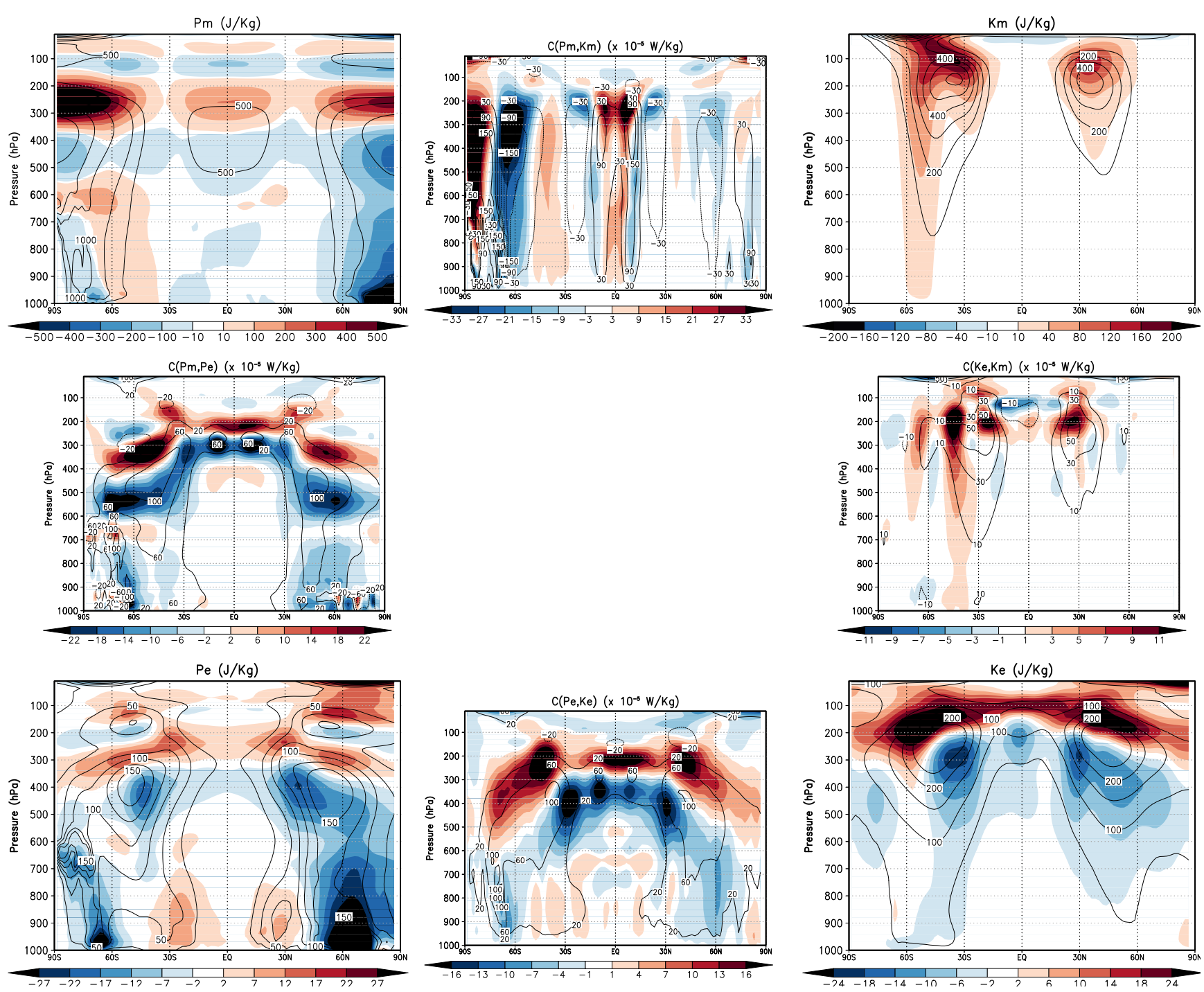

Fig. 3. Vertical cross-sections of the 4-box LEC terms for the $1 \times \mathrm{CO}_{2}$ control experiment (contours), and their change in the $2 \times \mathrm{CO}_{2}$ experiment (color shaded). Counterclockwise, starting from the upper left: $P_{\mathrm{m}}, C\left(P_{\mathrm{m}}, P_{\mathrm{e}}\right), P_{\mathrm{e}}, C\left(P_{\mathrm{e}}, K_{\mathrm{e}}\right), K_{\mathrm{e}}, C\left(K_{\mathrm{e}}, K_{\mathrm{m}}\right), K_{\mathrm{m}}$, and $C\left(P_{\mathrm{m}}, K_{\mathrm{m}}\right)$. Units are $\mathrm{J} \mathrm{kg}^{-1}$ for reservoirs, and $10^{-5} \mathrm{~W} \mathrm{~kg}^{-1}$ for conversion terms.

experiments (Fig. 5, upper panel): $P_{\text {te }}$ is almost 3 times as large as $P_{\text {se }}$, and $K_{\text {te }}$ is almost 7 times as large as $K_{\text {se }}$. We find similar ratios between $C\left(P_{\mathrm{m}}, P_{\mathrm{te}}\right), C\left(P_{\mathrm{te}}, K_{\mathrm{te}}\right)$, $C\left(K_{\mathrm{te}}, K_{\mathrm{m}}\right)$ and $C\left(P_{\mathrm{m}}, P_{\mathrm{se}}\right), C\left(P_{\mathrm{se}}, K_{\mathrm{se}}\right), C\left(K_{\mathrm{se}}, K_{\mathrm{m}}\right)$, respectively. The stationary eddy terms have smaller contributions, as expected. We find the same predominance of the transient eddy terms over the stationary eddy terms in two of the conversion rates regarding the $2 \times \mathrm{CO}_{2}$ energetics response (Fig. 5, lower panel): the responses of $C\left(P_{\mathrm{m}}, P_{\mathrm{e}}\right)$ and $C\left(K_{\mathrm{e}}, K_{\mathrm{m}}\right)$ are clearly dominated by the changes in $C\left(P_{\mathrm{m}}, P_{\mathrm{te}}\right)$ and $C\left(K_{\mathrm{te}}, K_{\mathrm{m}}\right)$, respectively. Only in the conversion term $C\left(P_{\mathrm{e}}, K_{\mathrm{e}}\right)$ we observe the global response similarly distributed in both $C\left(P_{\mathrm{se}}, K_{\mathrm{se}}\right)$ and $C\left(P_{\mathrm{te}}, K_{\mathrm{te}}\right)$. Both terms decrease by $0.02 \mathrm{~W} \mathrm{~m}^{-2}$, which would suggest that both stationary and transient eddies contribute equally to the most important conversion rate, $C\left(P_{\mathrm{e}}, K_{\mathrm{e}}\right)$. However, the fact that only this term shows this feature and not the other conversion rates seems somehow inconsistent. By looking at the vertical cross-sections of these terms we will be able to understand this apparent inconsistency, and determine if the stationary eddy components have an important contribution to the main energetics response.

In terms of the vertical cross-sections of the decomposed reservoirs (Fig. 6) the pattern of increase in the upper troposphere and decrease below is mainly due to the response of the transient components $P_{\text {te }}$ and $K_{\text {te }}$. The main pattern of change of $P_{\mathrm{e}}$ to which we have referred to, comes from the pattern of change of $P_{\mathrm{te}}$. The pattern of change of $P_{\text {se }}$ reveals some particular features, but these do not contribute to the main global energetics response. We deal with 

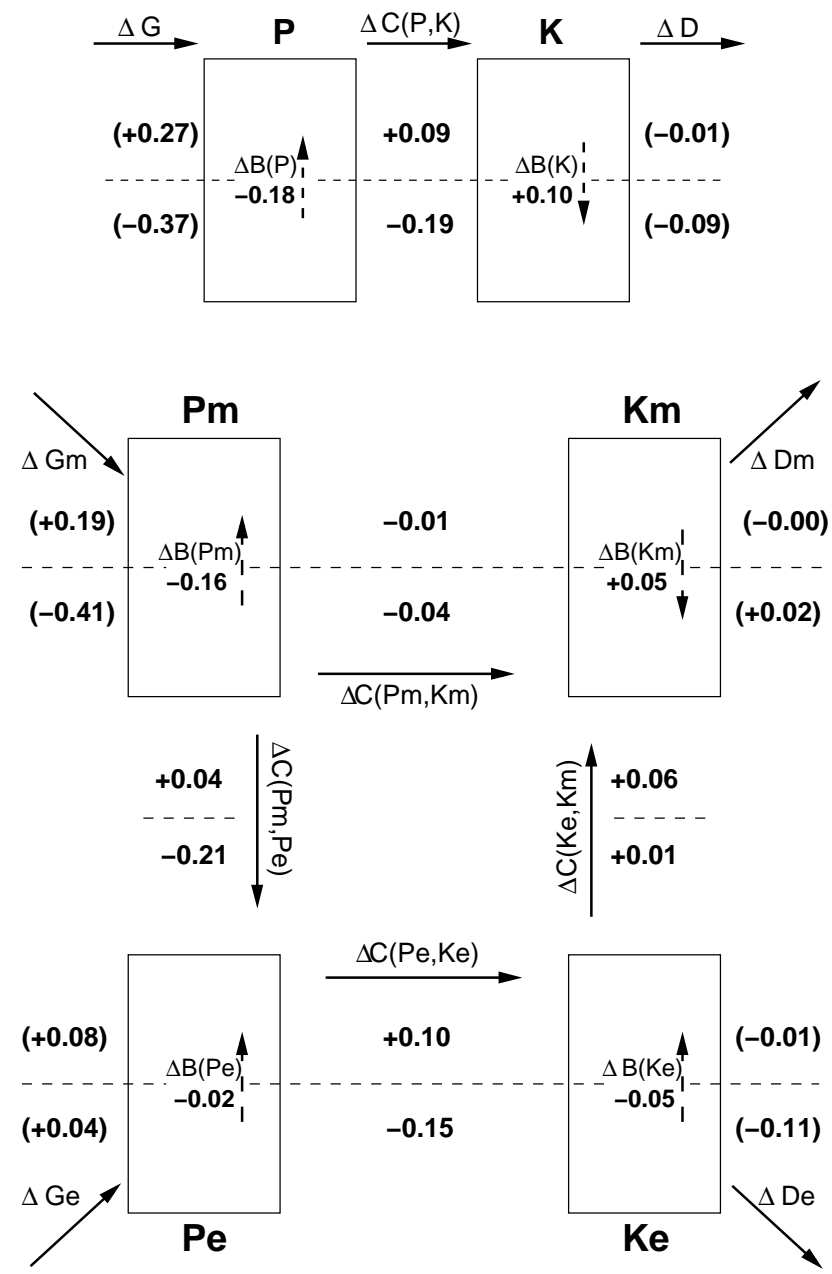

Fig. 4. 2-box (above) and 4-box (below) LEC diagrams of the changes in energy generation, conversion and dissipation rates due to $\mathrm{CO}_{2}$ doubling. Units are $\mathrm{W} \mathrm{m}^{-2}$. Arrows indicate the direction corresponding to positive values; negative values imply opposite direction.

these in the next subsection. Regarding $K_{\mathrm{e}}$, the main upperstrengthening and lower-weakening response is coming from the $K_{\text {te }}$ response. The contribution of the change of $K_{\text {se }}$ to the $K_{\mathrm{e}}$-response is much smaller (note the different scale used in the plots of $K_{\mathrm{se}}$ and $K_{\mathrm{te}}$ ).

By looking at the vertical cross-sections of the conversion rates (Fig. 7), we can also see that the response to a $\mathrm{CO}_{2}-$ doubling is dominated by the response of the conversions related to the transient eddy reservoirs. Clearly, the patterns of change of $C\left(P_{\mathrm{m}}, P_{\mathrm{te}}\right), C\left(P_{\mathrm{te}}, K_{\mathrm{te}}\right)$, and $C\left(K_{\mathrm{te}}, K_{\mathrm{m}}\right)$ are very similar to the patterns of change of $C\left(P_{\mathrm{m}}, P_{\mathrm{e}}\right), C\left(P_{\mathrm{e}}, K_{\mathrm{e}}\right)$ and $C\left(K_{\mathrm{e}}, K_{\mathrm{m}}\right)$ (Fig. 3). We expected this from $C\left(P_{\mathrm{m}}, P_{\mathrm{te}}\right)$ and $C\left(K_{\mathrm{te}}, K_{\mathrm{m}}\right)$, because their globally integrated values were larger than the ones of their corresponding stationary eddy terms. However, this was not so clear for $C\left(P_{\mathrm{e}}, K_{\mathrm{e}}\right)$. We had pointed out that the changes in the globally integrated values of $C\left(P_{\mathrm{te}}, K_{\mathrm{te}}\right)$ and $C\left(P_{\mathrm{se}}, K_{\mathrm{se}}\right)$ are very similar (both

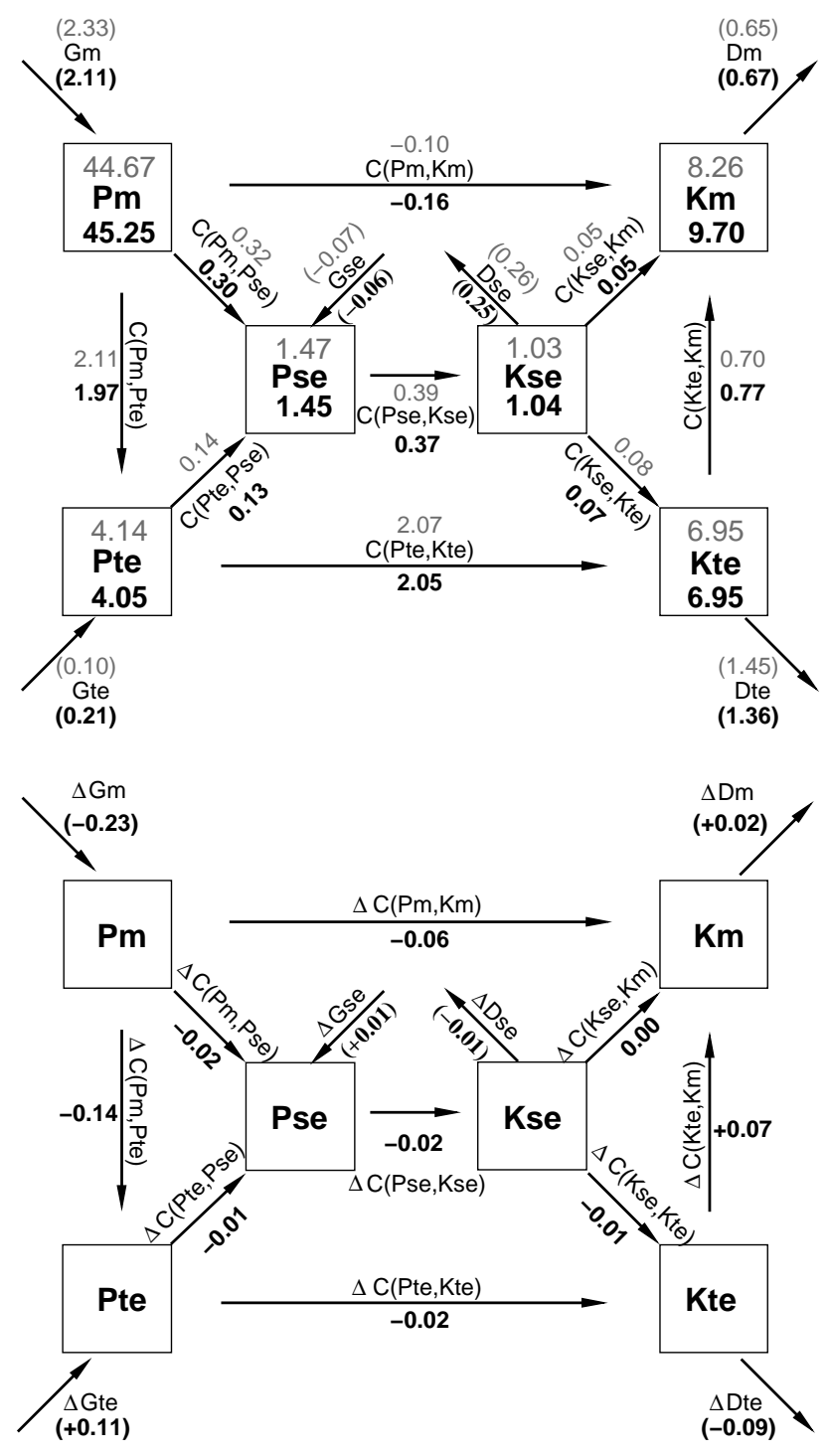

Fig. 5. 6-box LEC diagram (after transient and stationary eddy decomposition). Upper panel shows values for the $1 \times \mathrm{CO}_{2}$ (above, gray) and $2 \times \mathrm{CO}_{2}$ (below, black) experiments. Generation and dissipation rates (in parenthesis) are obtained as residuals. Lower panel shows the $2 \times \mathrm{CO}_{2}$ changes in energy generation, conversion and dissipation rates. Units are $10^{5} \mathrm{~J} \mathrm{~m}^{-2}$ for reservoirs and $\mathrm{W} \mathrm{m}^{-2}$ for conversion, generation and dissipation terms. Arrows indicate the direction corresponding to positive values; negative values imply opposite direction.

decrease by $0.02 \mathrm{~W} \mathrm{~m}^{-2}$ ). However, looking at the vertical cross-sections of these two conversion rates, we clearly see that the pattern of change that determines $C\left(P_{\mathrm{e}}, K_{\mathrm{e}}\right)$ 's vertical cross-section (Fig. 3) comes from $C\left(P_{\mathrm{te}}, K_{\mathrm{te}}\right)$ and not from $C\left(P_{\mathrm{se}}, K_{\mathrm{se}}\right)$. But because this pattern consists of a strengthening in the upper-troposphere and a weakening below, its total change is very small, and happens to be similar to the total change of $C\left(P_{\mathrm{se}}, K_{\mathrm{se}}\right)$. Thus, the vertical 


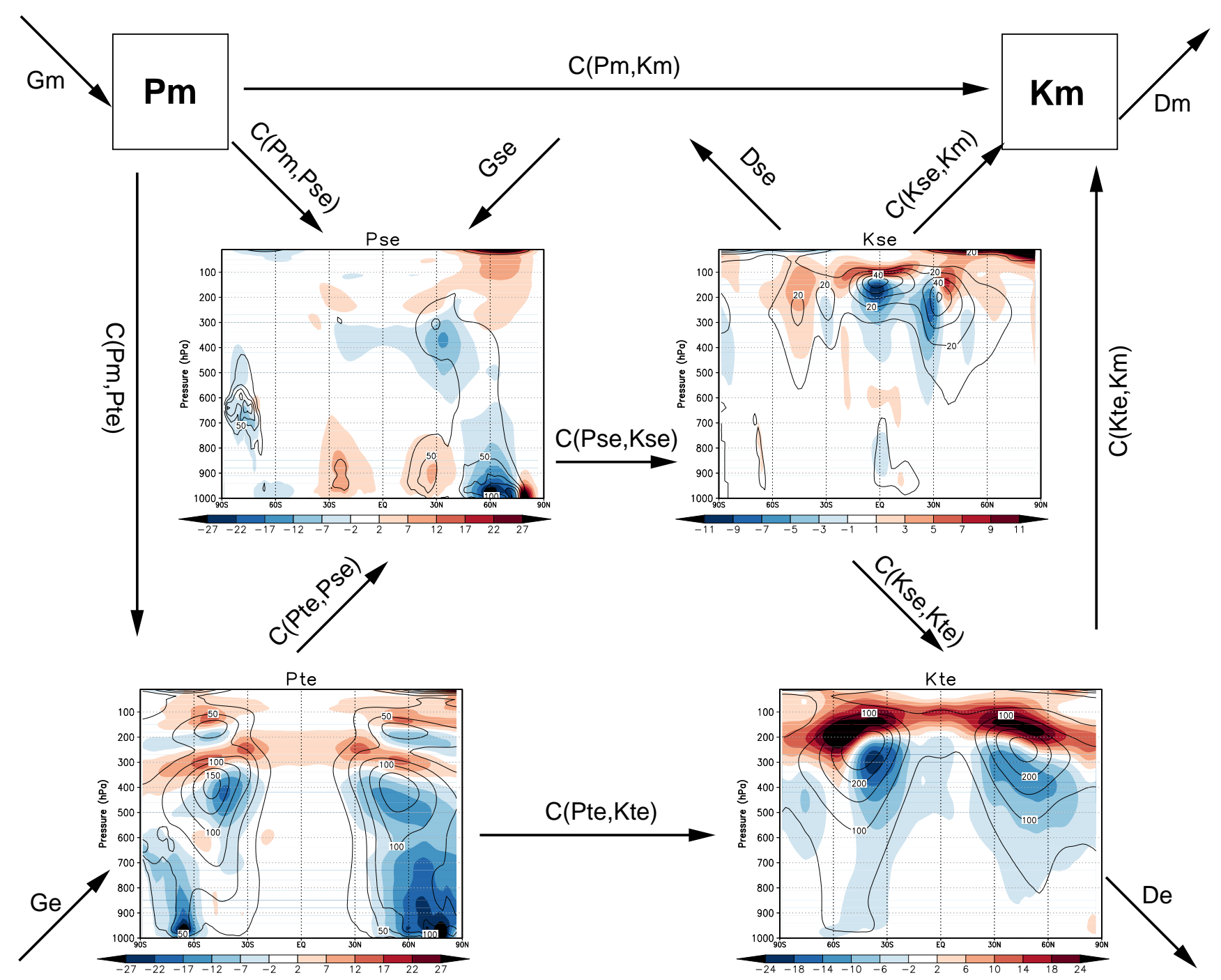

Fig. 6. Vertical cross sections of the stationary and transient eddy reservoirs $\left(P_{\mathrm{se}}, P_{\mathrm{te}}, K_{\mathrm{se}}\right.$ and $\left.K_{\mathrm{te}}\right)$ for the $1 \times \mathrm{CO}_{2}$ control experiment (contours), and their $2 \times \mathrm{CO}_{2}$ change (color shaded). Units are $\mathrm{J} \mathrm{kg}^{-1}$.

cross-sections reveal that the main energetics response is taking place via the transient eddies, i.e., via $C\left(P_{\mathrm{te}}, K_{\mathrm{te}}\right)$, and not via $C\left(P_{\mathrm{se}}, K_{\mathrm{se}}\right)$.

Summing up, the transient eddy reservoirs and conversion rates clearly dominate the global energetics response. This means that the main energetics response takes place along the path $P_{\mathrm{m}} \rightarrow P_{\mathrm{te}} \rightarrow K_{\mathrm{te}} \rightarrow K_{\mathrm{m}}$. The energetics response to doubling of $\mathrm{CO}_{2}$ concentrations that we have described in the previous section, as well as the one described by HDvS, corresponds to the response of the transient eddy components, whereas the stationary components have a very small contribution. In the following subsection we analyse the main cause for the response of this transient eddy response based on the effects of the zonal-mean warming pattern.

\subsubsection{The transient eddy response}

The main energetics response to a $\mathrm{CO}_{2}$ doubling consists of a strengthening of the LEC in the upper troposphere and a weakening below (HDvS). We now know that this dual response concerns the transient eddy components and not the stationary ones. Furthermore, HDvS relate this response to the zonal-mean warming pattern, finding that changes in meridional temperature gradient and mean static stability can explain, at least qualitatively, this dual response. We will now carry out a similar analysis with our higher resolution results, having in mind that this main response concerns the transient eddy components.

Following Held (1993), the dual energetics response could be related to the two main features of the zonal-mean warming pattern (Fig. 2). First, the tropical upper-tropospheric 


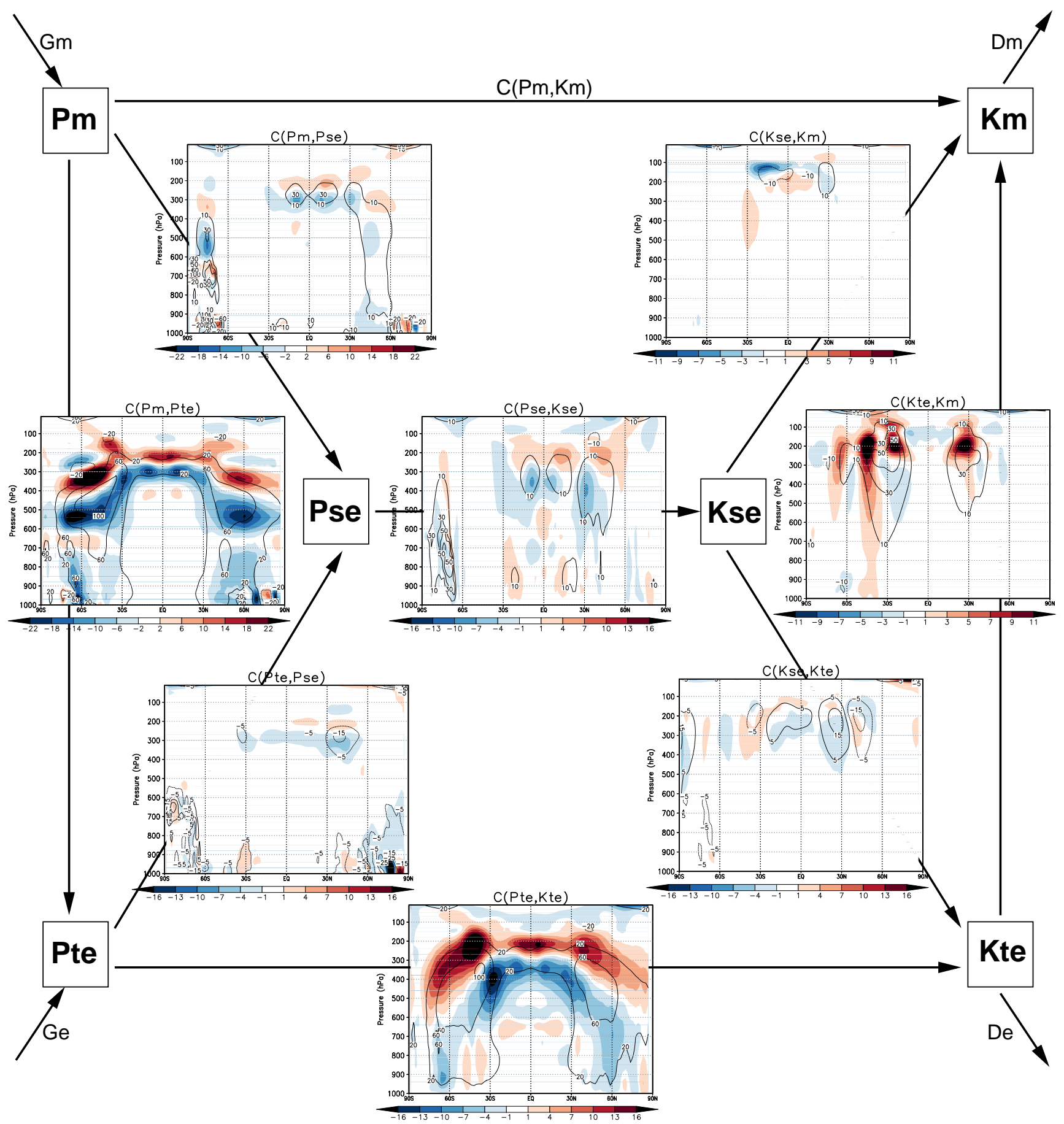

Fig. 7. Vertical cross sections of the stationary and transient eddy conversion terms $\left(C\left(P_{\mathrm{m}}, P_{\mathrm{te}}\right), C\left(P_{\mathrm{m}}, P_{\mathrm{se}}\right), C\left(P_{\mathrm{te}}, P_{\mathrm{se}}\right), C\left(P_{\mathrm{te}}, K_{\mathrm{te}}\right)\right.$, $C\left(P_{\mathrm{se}}, K_{\mathrm{se}}\right) C\left(K_{\mathrm{te}}, K_{\mathrm{m}}\right)$, and $\left.C\left(K_{\mathrm{te}}, K_{\mathrm{m}}\right)\right)$ for the $1 \times \mathrm{CO}_{2}$ control experiment (contours), and their $2 \times \mathrm{CO}_{2}$ change (color shaded). Units are $10^{-5} \mathrm{~W} \mathrm{~kg}^{-1}$.

warming implies an increase of the meridional temperature gradient in the upper-troposphere and would therefore cause more baroclinicity. Hence, a strengthening of the LEC. Second, the high-latitude surface warming reduces the meridional temperature gradient and by doing so, it decreases baroclinicity. This would imply a weakening of the LEC.
Nevertheless, to obtain a complete picture of the possible effects of the warming pattern on the energetics response, changes in the stratification, i.e., in mean static stability, should be taken into account. Mean static stability appears in various LEC-terms in the form of inverse static stability, $\gamma$. From the point of view of static stability changes, 
the warming pattern would cause exactly the opposite effects than the ones described above: the tropical uppertropospheric warming increases mean static stability, weakening the generation rate of available potential energy, as well as available potential energy (because $\gamma$ would decrease). The high-latitude surface warming would cause an increase of $G_{\mathrm{m}}$ and $P_{\mathrm{m}}$, and therefore a strengthening of the LEC, because it implies a weakening of mean static stability.

Following HDvS, and in order to try to assess how these different effects of the warming pattern combine in order to produce the $2 \times \mathrm{CO}_{2}$ energetics response, we look here into the vertical profile of the changes in $\gamma$, the inverse mean static stability (Fig. 8, upper panel) and in $P_{\mathrm{m}}$ (Fig. 8, lower panel). The vertical profile of $P_{\mathrm{m}}$ decreases strongly near the surface (by roughly $10 \%$ ), whereas $\gamma$ does not change much there. This suggests that this feature cannot be caused by changes in $\gamma$, but rather by the reduced horizontal temperature variance due to the high-latitude surface warming. On the other hand, the decrease of $P_{\mathrm{m}}$ above $600 \mathrm{hPa}$ is clearly driven by the relative decrease of $\gamma$, i.e., the increase in global mean static stability. We can conclude this because in this region (a) the relative changes of both $\gamma$ and $P_{\mathrm{m}}$ are similar, and (b) the warming is rather homogeneous latitudinally so that very little changes in meridional temperature gradient take place. In between these two regions, above the surface and below $600 \mathrm{hPa}$, the resulting change in $P_{\mathrm{m}}$ is likely to be due to a combination of both effects: the decrease of $\gamma$ (increased mean static stability) and the reduced meridional temperature gradient near the surface. Above $250 \mathrm{hPa}, P_{\mathrm{m}}$ reaches an increase of about $140 \%$ (out of scale in Fig. 8), which must be related to horizontal temperature variance changes, because the relative changes of $\gamma$ are never as large.

We assume that the changes in $P_{\mathrm{m}}$ reflect to a certain extent the response of the whole LEC, for changes in $P_{\mathrm{m}}$ are expected to indicate corresponding changes in baroclinicity. Furthermore, we have found that the largest changes due to $\mathrm{CO}_{2}$ doubling are found in $G_{\mathrm{m}}$, the generation rate of $P_{\mathrm{m}}$. Actually, understanding the response of $G_{\mathrm{m}}$ would explain the whole energetics response from the point of view of the LEC without invoking the concept of baroclinicity. This is because under steady state conditions, changes in the generation rates must be balanced by corresponding changes in the conversion and dissipation rates, hence in the whole energetics. On the contrary, changes in reservoirs are not necessarily balanced by changes in other terms. Unfortunately, we cannot calculate profiles of the generation rates because we only obtain these as residuals of the globally integrated terms. Nevertheless, the changes in the profiles of $P_{\mathrm{m}}$ can give us a good idea about the changes in the profiles of $G_{\mathrm{m}}$. First of all, because the expression for $G_{\mathrm{m}}$ (Eq. A16) is very similar to the expression for $P_{\mathrm{m}}$ (Eq. A1). It is also proportional to $\gamma$, so changes in mean static stability should affect $G_{\mathrm{m}}$ in a similar way as they affect $P_{\mathrm{m}}$. The difference with $P_{\mathrm{m}}$ is that instead of being proportional to $[\langle T\rangle]^{\prime \prime}[\langle T\rangle]^{\prime \prime}$, the horizontal variance of temperature, it is proportional to $[\langle T\rangle]^{\prime \prime}[\langle Q\rangle]^{\prime \prime}$,
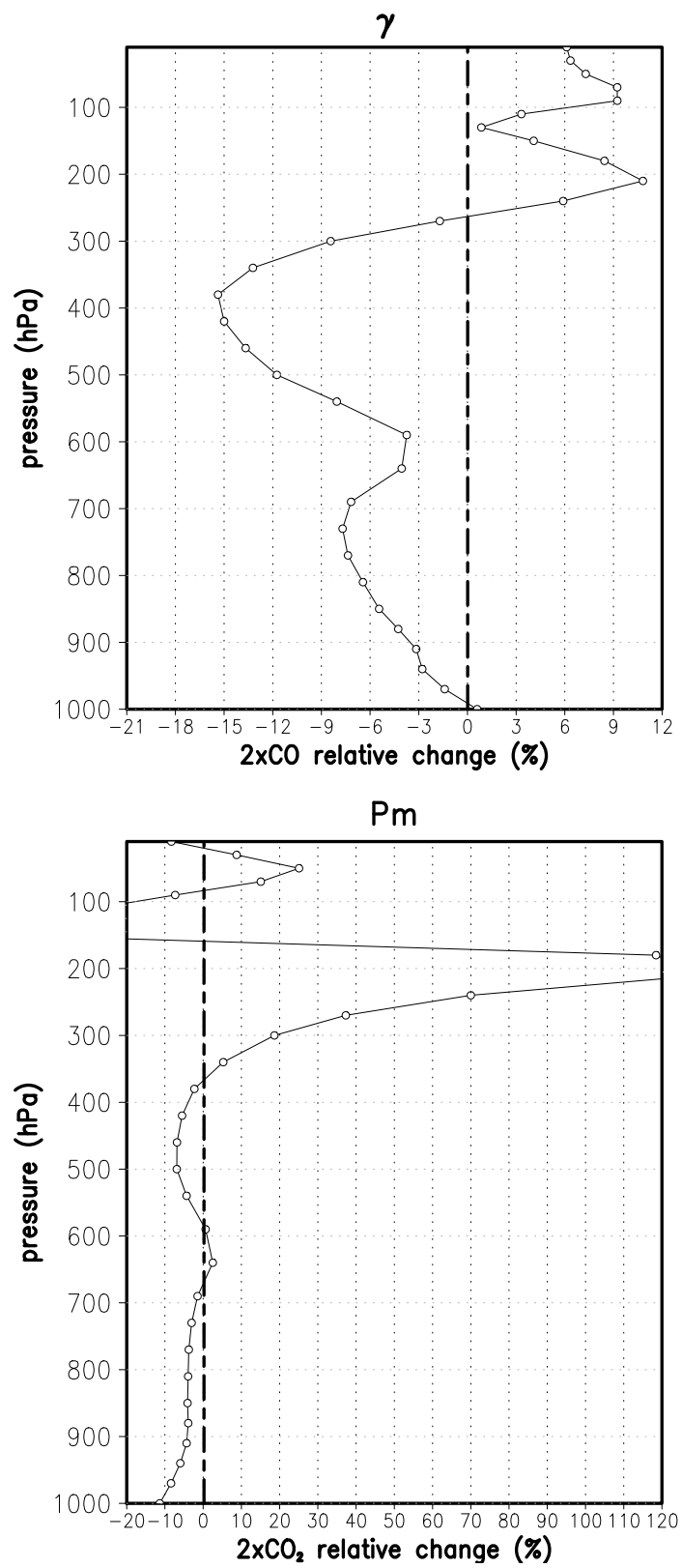

Fig. 8. Relative change of the vertical profile of gamma, $\gamma$ (above) and of $P_{\mathrm{m}}$ (below) when doubling $\mathrm{CO}_{2}$ concentrations (difference divided by the $1 \times \mathrm{CO}_{2}$ value).

the "correlation" between deviations of temperature and diabatic heating. In other words, $G_{\mathrm{m}}$ has positive contributions from relatively warm latitudes that have net diabatic heating, or from relatively cold latitudes that have net diabatic cooling. Negative contributions would imply relatively warm latitudes with net diabatic cooling, or relatively cold latitudes with net diabatic heating. We know that on average, the relatively warm latitudes - the low latitudes - have an excess of diabatic heating, while the relatively cold latitudes the high latitudes - have an excess of diabatic cooling (e.g., 
Peixoto and Oort, 1992). Hence, $G_{\mathrm{m}}$ is by far positive, because $[\langle T\rangle]^{\prime \prime}$ and $[\langle Q\rangle]^{\prime \prime}$ are strongly correlated. This is not the case of $G_{\mathrm{e}}$, for example. In terms of deviations from the time and zonal-means, there is no such strong correlation between $\langle T\rangle^{*}$ and $\langle Q\rangle^{*}$, or between $T^{\prime}$ and $Q^{\prime}$. Most of the processes leading to the generation of $P_{\mathrm{e}}$ cancel each other, such that globally, this term ends up being very close to zero, or even slightly negative (Lorenz, 1955; Romanski, 2009).

The fact that $[\langle T\rangle]^{\prime \prime}$ and $[\langle Q\rangle]^{\prime \prime}$ are so highly correlated implies that $G_{\mathrm{m}}$ should have a distribution similar to $P_{\mathrm{m}}$. The vertical profile of $G_{\mathrm{m}}$ should have characteristics very similar to those of $P_{\mathrm{m}}$, although with different units. We do not find any reason for this high correlation to change significantly in a $2 \times \mathrm{CO}_{2}$ climate. The pattern of change of $G_{\mathrm{m}}$ must be very similar to the pattern of change of $P_{\mathrm{m}}$. Therefore, we can extend our analysis regarding $P_{\mathrm{m}}$ to $G_{\mathrm{m}}$ : having in mind that $G_{\mathrm{m}}$ should behave in a very similar way to $P_{\mathrm{m}}$, we can conclude that the increase in $G_{\mathrm{m}}$ in the upper region is related to the strong increase in horizontal temperature variance due to the tropical upper-tropospheric warming. This assumes that in this upper region, the correlation between $[\langle T\rangle]^{\prime \prime}$ and $[\langle Q\rangle]^{\prime \prime}$ becomes stronger due to the larger meridional temperature gradient. The decrease of $G_{\mathrm{m}}$ in the lower region is related to a combination of the increased mean static stability due to the upper tropospheric warming, and the decreased meridional temperature gradient due to the high-latitude surface warming, which should decrease the correlation between $[\langle T\rangle]^{\prime \prime}$ and $[\langle Q\rangle]^{\prime \prime}$ near the surface. This dual response of $G_{\mathrm{m}}$ drives then the strengthening of the LEC in the upper region and the weakening in the lower region. This result is fully consistent with the conclusions obtained by HDvS, but knowing now that these concern the transient eddy response.

\subsubsection{The stationary eddy response}

Finally, even though the stationary eddy response is not so relevant for the main energetics response, $P_{\text {se }}$ shows some features that are worth analysing. When doubling $\mathrm{CO}_{2}$ concentrations, $P_{\mathrm{se}}$ increases in two regions near the surface at around $25^{\circ} \mathrm{N}$ and $25^{\circ} \mathrm{S}$, which contribute to the vertical cross-section of $P_{\mathrm{e}}$ in Fig. 3. The distribution of the change in the integrand of $P_{\text {se }}$ at $910 \mathrm{hPa}$ (Fig. 9, upper panel), calculated as the change in $\left(c_{\mathrm{p}} / 2\right) \gamma\langle\beta\rangle\langle T\rangle^{* 2}$, reveals the causes for these features. The increase region around $25^{\circ} \mathrm{S}$ is due to an increase in $P_{\text {se }}$ over Australia, central-South America and a region over the Eastern Pacific, and the increase region around $25^{\circ} \mathrm{N}$ is related to an increase in $P_{\text {se }}$ over the Saharan and the Arabian Deserts. These features are clearly related to the $2 \times \mathrm{CO}_{2}$ temperature change in these regions, and in particular, to the changes in the zonal anomalies of temperature, $\langle T\rangle^{*}$ (Fig. 9, lower panel). The regions that are causing changes in $P_{\mathrm{se}}$ correspond to regions where the warming pattern is such as to enhance the zonal anomalies of the temperature field. For example, the Australian continent has a positive contribution to $\langle T\rangle^{*}$ in the $1 \times \mathrm{CO}_{2}$ case (contour lines in Fig. 9, lower panel), i.e., it is warmer than the zonal-mean for this latitude belt. The strong positive change of $\langle T\rangle^{*}$ in this region indicates that this zonal anomaly is enhanced under $2 \times \mathrm{CO}_{2}$ conditions. The same happens over the Saharan and Arabian deserts, where positive values of $\langle T\rangle^{*}$ are enhanced when doubling $\mathrm{CO}_{2}$ concentrations. On the other hand, the Eastern Pacific has a lower mean temperature than its zonal belt, which reflects on a negative value of $\langle T\rangle^{*}$ in the $1 \times \mathrm{CO}_{2}$ case (dashed contour lines in Fig. 9, lower panel). This zonal anomaly is also enhanced when doubling $\mathrm{CO}_{2}$ concentrations, because it coincides with a negative change in $\langle T\rangle^{*}$. Therefore, $P_{\mathrm{se}}$ increases here as well.

The opposite happens in the Atlantic between Greenland and Northern Europe. This region is on average warmer than its latitudinal-belt, causing the largest contribution to $P_{\text {se }}$ globally. This is also reflected on the strong positive values of $\langle T\rangle^{*}$ in the $1 \times \mathrm{CO}_{2}$ case (contour lines in Fig. 9, below). The $\langle T\rangle^{*}$-change is negative in this region, so that this zonal anomaly becomes weaker under $2 \times \mathrm{CO}_{2}$ conditions. Hence $P_{\text {se }}$ decreases strongly in this region. This happens because (a) the continental warming is stronger, and (b) the weakening of the Atlantic meridional overturning reduces the sea surface temperature in this region (Meehl et al., 2007).

In other words, there is a smaller land-sea contrast at the northern high latitudes, whereas the opposite is true for the subtropics, around $25^{\circ} \mathrm{N}$ and $25^{\circ} \mathrm{S}$, where the land-sea contrasts are enhanced. These features of the $2 \times \mathrm{CO}_{2}$ warming pattern are not new. Most current climate models predict such a warming pattern (Meehl et al., 2007). Such a warming pattern cause changes in $P_{\text {se }}$ that stand out and are directly related to the $2 \times \mathrm{CO}_{2}$ warming, but they do not affect the global energetics response of the atmosphere. In order to do so, these changes in $P_{\mathrm{se}}$ would also have to cause changes in the conversion rate into $K_{\mathrm{se}}, C\left(P_{\mathrm{se}}, K_{\mathrm{se}}\right)$. There is indeed a contribution around $25^{\circ} \mathrm{N}$ and $25^{\circ} \mathrm{S}$ in this conversion rate, but it is very small. Actually, we expect this conversion to be small in these regions, because in order to convert $P_{\text {se }}$ into $K_{\mathrm{se}}$, a good correlation between $\langle\omega\rangle^{*}$ and $\langle\alpha\rangle^{*}$ is needed (see Appendix A). Intuitively, this can be seen as rising of relatively warm air and sinking of relatively cold air in the stationary eddies. However, these regions are mostly subtropical deserts where there is relatively warm air, but very little or no rising of air. Therefore, $C\left(P_{\mathrm{se}}, K_{\mathrm{se}}\right)$ has a very small contribution from these regions, and the global energetics is not affected much by such features in $P_{\text {se }}$. Regarding the decrease of $P_{\mathrm{se}}$ in the North Atlantic region, this does not have any effect on the conversion rate $C\left(P_{\mathrm{se}}, K_{\mathrm{se}}\right)$ either. In this case, the only reason we find is that the contribution of $C\left(P_{\mathrm{se}}, K_{\mathrm{se}}\right)$ to the total $C\left(P_{\mathrm{e}}, K_{\mathrm{e}}\right)$ in this region is so small already that a further reduction in the reservoir of $P_{\mathrm{se}}$ makes no significant difference. 

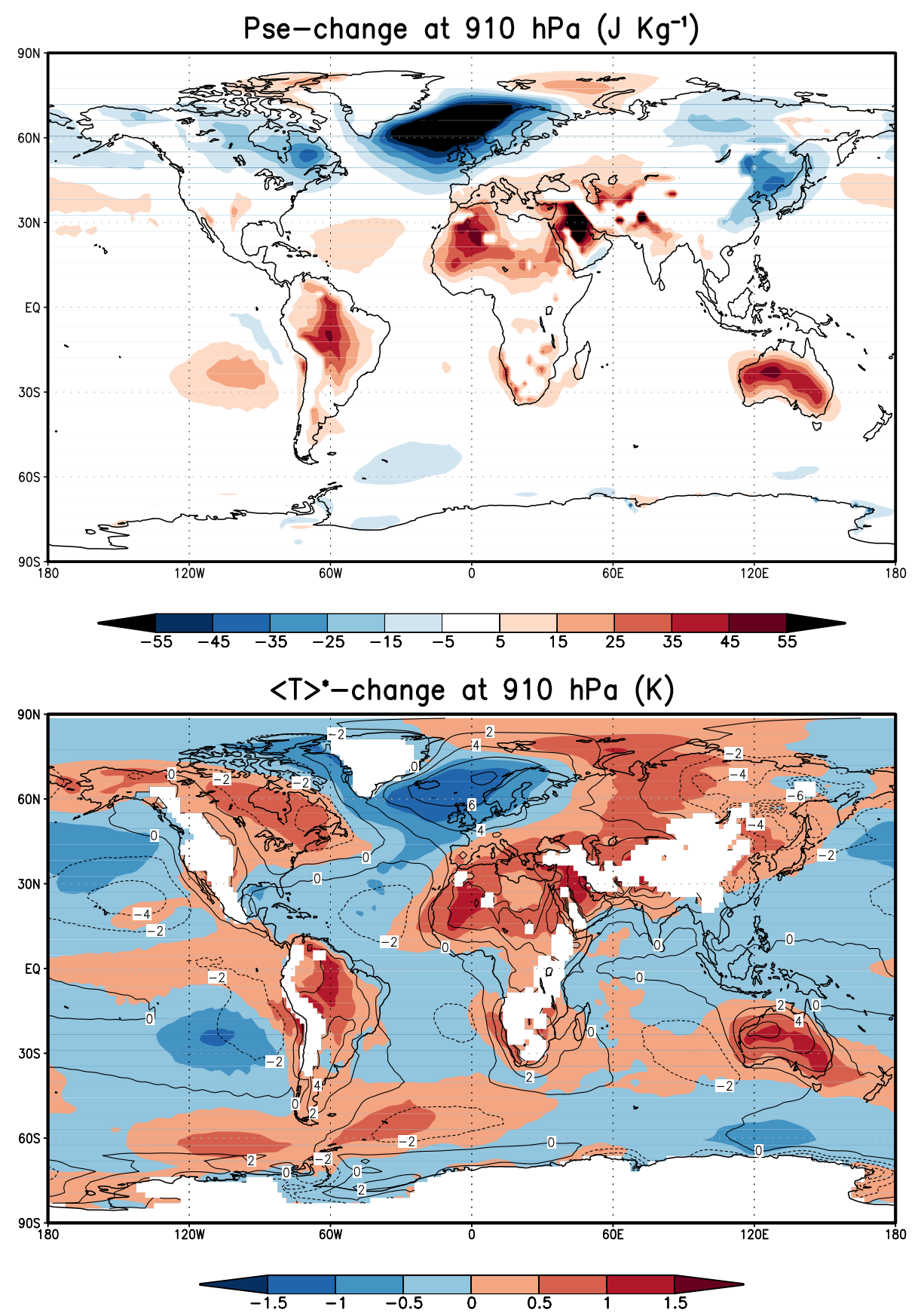

Fig. 9. $P_{\mathrm{Se}}$-change (upper panel) and $\langle T\rangle^{*}$-change, the changes in the zonal anomalies of temperature (lower panel) at $910 \mathrm{hPa}$ due to $\mathrm{CO}_{2}$ doubling. Contour lines in lower panel indicate the $1 \times \mathrm{CO}_{2}$ distribution of $\langle T\rangle^{*}$.

\section{Conclusions and discussion}

We use here the Lorenz Energy Cycle (LEC) in order to estimate the global energetic activity of the atmosphere in the coupled atmosphere-ocean ECHAM5/MPI-OM model. Using two equilibrium runs $\left(1 \times \mathrm{CO}_{2}\right.$ and $\left.2 \times \mathrm{CO}_{2}\right)$ we estimate the response of the energetic activity due to doubling of $\mathrm{CO}_{2}$ concentrations in the same way as HDvS have done with a coarser resolution version of the same model. The main $2 \times \mathrm{CO}_{2}$ energetics response that we find here is fully consistent with the coarser resolution results (HDvS). We find a $4 \%$ weakening of the global energetic activity when doubling $\mathrm{CO}_{2}$ concentrations, as measured by the change in the total $P$-to- $K$ conversion rate. This result is also consistent with other studies (Boer, 1995; Marquet, 2006; Lucarini, 2009), which mainly attribute this overall weakening to the reduction in pole-to-equator temperature gradient and to weaker land-sea contrasts during the winter season. We 
also find that this overall weakening results from a dual response: a strengthening of the energetic activity in the uppertroposphere, and a weakening in the lower and middle troposphere that dominates the globally integrated response. Note that the global weakening in the LEC-strength implies as well a decrease in the global dissipation rate of kinetic energy, which is often assumed to be related to weaker surface winds (Lucarini et al., 2010). However, our experiments do not show a significant global weakening of the near-surface winds (not shown here). Further research would be needed to determine the direct causes for the weakening of the global dissipation rate.

Having verified that the main energetic response is fully consistent with the coarser resolution version of the coupled model (HDvS), we perform an additional decomposition of the eddy reservoirs of the LEC in order to determine how the stationary and the transient eddies contribute to the energetics response. As expected (Oort and Peixoto, 1974; Holton, 2004), we find that most of the energetic activity originates from the transient eddies. However, this is not necessarily the case when referring to the $2 \times \mathrm{CO}_{2}$ response. In principle, the response of the stationary eddy components could also have a significant contribution to the main energetics response. Nevertheless, we find that the main energetics response is determined by the response of the transient eddy reservoirs $P_{\text {te }}$ and $K_{\text {te }}$ and by the corresponding energy conversion rates, $C\left(P_{\mathrm{m}}, P_{\mathrm{te}}\right), C\left(P_{\mathrm{te}}, K_{\mathrm{te}}\right)$ and $C\left(K_{\mathrm{te}}, K_{\mathrm{m}}\right)$. The response of the stationary eddy reservoirs $P_{\text {se }}$ and $K_{\text {se }}$, and of their corresponding energy conversion terms, $C\left(P_{\mathrm{m}}, P_{\mathrm{se}}\right)$, $C\left(P_{\mathrm{te}}, P_{\mathrm{se}}\right), C\left(P_{\mathrm{se}}, K_{\mathrm{se}}\right), C\left(K_{\mathrm{se}}, K_{\mathrm{te}}\right)$, and $C\left(K_{\mathrm{se}}, K_{\mathrm{m}}\right)$, is very small compared to the transient component response. Therefore, the main energetics response we describe above arises mainly from the transient eddy components.

By analysing the zonal-mean warming pattern together with the $P_{\mathrm{m}}$-response, it is possible to explain qualitatively the dual energetics response, which we now know to be related to the transient eddy terms. The strengthening in the upper-troposphere is caused by the increased meridional temperature gradient due to the tropical upper-tropospheric warming, while the weakening below is due to a combination of a weaker meridional temperature gradient - caused by the high-latitude surface warming - and an enhanced mean static stability - caused by the tropical upper-tropospheric warming. This shows how relevant the thermal structure of the atmosphere is for the energetics response, in agreement with previous studies (see, e.g., O'Gorman and Schneider, 2008). Further, our results indicate that static stability effects may be more important than previously thought, in accordance with other findings by O'Gorman (2010). In particular, static stability changes tend to counteract the expected response due to meridional temperature gradient changes. In order to completely separate the effects of the different features of the warming pattern on the energetics of the atmosphere, additional simulations are carried out in which specific atmospheric warming patterns are forced. These experiments, to be reported elsewhere, are expected to verify the current results presented here, and in particular, to quantify the relative contribution of the tropical upper-tropospheric warming and the high-latitude surface warming to the dual energetics response of the LEC.

Regarding the stationary eddy components, the only term that shows some outstanding features is $P_{\text {se }}$. Although these do not affect the main energetics response, they stand out and are related to regional warming patterns due to the $\mathrm{CO}_{2}-$ doubling. $\quad P_{\text {se }}$ increases over two regions near the surface symmetric about the equator, around $25^{\circ} \mathrm{N}$ and $25^{\circ} \mathrm{S}$. This increase is mainly caused by the stronger warming of subtropical deserts relative to their corresponding zonal belt, enhancing the temperature contrasts that contribute to $P_{\mathrm{se}}$ in these regions. Furthermore, there is also a strong decrease in $P_{\text {se }}$ near the surface around $60^{\circ} \mathrm{N}$, which is related to the weaker warming of the North Atlantic with respect to this latitude belt (i.e., stronger warming of the continents in comparison to the North Atlantic). All these regional warming features are well known from different climate models (Meehl et al., 2007). We find here that these warming patterns have a clear impact on the response of $P_{\text {se }}$. However, they do not affect the main global energetics response, which is driven by the response of the transient eddy components of the LEC.

\section{Appendix A}

\section{Lorenz Energy Cycle terms}

The Lorenz Energy Cycle (LEC) terms are given as integrals over the atmosphere. To avoid using extrapolated values for points below the surface in the integrals, we use the $\beta$ function proposed by Boer (1982) and defined as $\beta=0$ for $p>p_{\mathrm{s}}$, and $\beta=1$ for $p \leq p_{\mathrm{s}}$, where $p$ is pressure and $p_{\mathrm{s}}$ is surface pressure. This $\beta$ function is not only a weighting factor in the final expressions, but is also used to weight each zonal and time mean, as described in detail by Boer (1982). The expressions we use here are equivalent to the ones used by HDvS, but separating the transient and stationary eddies. The symbols we use are listed in Table A1.

The reservoirs of the LEC are given by:

$$
\begin{aligned}
& P_{\mathrm{m}}=\frac{c_{\mathrm{p}}}{2} \int \gamma[\langle\beta\rangle][\langle T\rangle]^{\prime \prime 2} \rho \mathrm{d} V \\
& P_{\mathrm{se}}=\frac{c_{\mathrm{p}}}{2} \int \gamma\left[\langle\beta\rangle\langle T\rangle^{* 2}\right] \rho \mathrm{d} V \\
& P_{\mathrm{te}}=\frac{c_{\mathrm{p}}}{2} \int \gamma\left[\langle\beta\rangle\left\langle T^{\prime 2}\right\rangle\right] \rho \mathrm{d} V \\
& K_{\mathrm{m}}=\frac{1}{2} \int[\langle\beta\rangle]\left([\langle u\rangle]^{2}+[\langle v\rangle]^{2}\right) \rho \mathrm{d} V \\
& K_{\mathrm{se}}=\frac{1}{2} \int\left[\langle\beta\rangle\left(\langle u\rangle^{* 2}+\langle v\rangle^{* 2}\right)\right] \rho \mathrm{d} V
\end{aligned}
$$


Table A1. List of symbols.

\begin{tabular}{|c|c|}
\hline Symbol & Description \\
\hline$a$ & Earth's average radius \\
\hline$c_{\mathrm{p}}$ & specific heat at constant pressure \\
\hline $\mathrm{d} A$ & surface element \\
\hline $\mathrm{d} V$ & volume element \\
\hline$g$ & acceleration due to gravity \\
\hline$k$ & $R / c_{\mathrm{p}}$ \\
\hline$p$ & pressure \\
\hline$p_{\mathrm{S}}$ & surface pressure \\
\hline$t$ & time \\
\hline$u$ & zonal wind component \\
\hline$v$ & meridional wind component \\
\hline$z$ & geopotential height \\
\hline$B(X)$ & boundary flux of $X$ \\
\hline$C(X, Y)$ & conversion rate from $X$ to $Y$ \\
\hline$D(Y)$ & dissipation rate of $Y$ \\
\hline$F_{x}, F_{y}$ & frictional force in $x$ and $y$ \\
\hline$K_{\text {te }}$ & transient eddy kinetic energy \\
\hline$K_{\mathrm{se}}$ & stationary eddy kinetic energy \\
\hline$K_{\mathrm{e}}$ & eddy kinetic energy $\left(K_{\mathrm{te}}+K_{\mathrm{se}}\right)$ \\
\hline$K_{\mathrm{m}}$ & zonal mean kinetic energy \\
\hline$P_{\text {te }}$ & transient eddy available potential energy \\
\hline$P_{\mathrm{se}}$ & stationary eddy available potential energy \\
\hline$P_{\mathrm{e}}$ & eddy available potential energy $\left(P_{\mathrm{te}}+P_{\mathrm{se}}\right)$ \\
\hline$P_{\mathrm{m}}$ & zonal mean available potential energy \\
\hline$Q$ & diabatic heating rate \\
\hline$R$ & gas constant for dry air \\
\hline$T$ & temperature \\
\hline$\alpha$ & specific volume \\
\hline$\beta$ & equals 0 for underground grid points, 1 otherwise \\
\hline$\gamma$ & inverse mean static stability factor: $\frac{-\theta R}{T p c_{\mathrm{p}}}\left(\frac{\partial \tilde{\theta}}{\partial p}\right)^{-1}$ \\
\hline$\lambda$ & longitude \\
\hline$\phi$ & latitude \\
\hline$\rho$ & density \\
\hline$\theta$ & potential temperature \\
\hline$\omega$ & vertical velocity in isobaric coordinates \\
\hline
\end{tabular}

$\left.K_{\text {te }}=\frac{1}{2} \int\left[\langle\beta\rangle\left\langle u^{\prime 2}\right\rangle+\left\langle v^{\prime 2}\right\rangle\right)\right] \rho \mathrm{d} V$,

where $[X]$ denotes the zonal-mean of $X,\langle X\rangle$ the time mean of $X, \widetilde{X}$ the global mean over a constant pressure level, and $X^{*}, X^{\prime}$ and $X^{\prime \prime}$ are the corresponding deviations from these means.

The conversion rates are given by:

$$
\begin{array}{r}
C\left(P_{\mathrm{m}}, P_{\mathrm{se}}\right)=-c_{\mathrm{p}} \int \gamma\left[\langle\beta\rangle\langle v\rangle^{*}\langle T\rangle^{*}\right] \frac{\partial[\langle T\rangle]}{a \partial \phi} \rho \mathrm{d} V \\
-c_{\mathrm{p}} p^{-k}\left[\langle\beta\rangle\langle\omega\rangle^{*}\langle T\rangle^{*}\right] \frac{\partial\left(\gamma p^{\mathrm{k}}[\langle T\rangle]^{\prime \prime}\right)}{\partial p} \rho \mathrm{d} V
\end{array}
$$

$$
\begin{aligned}
& C\left(P_{\mathrm{m}}, P_{\mathrm{te}}\right)=-c_{\mathrm{p}} \int \gamma\left[\langle\beta\rangle\left\langle v^{\prime} T^{\prime}\right\rangle\right] \frac{\partial[\langle T\rangle]}{a \partial \phi} \rho \mathrm{d} V \\
& -c_{\mathrm{p}} \int p^{-k}\left[\langle\beta\rangle\left\langle\omega^{\prime} T^{\prime}\right\rangle\right] \frac{\partial\left(\gamma p^{\mathrm{k}}[\langle T\rangle]^{\prime \prime}\right)}{\partial p} \rho \mathrm{d} V \\
& C\left(P_{\mathrm{se}}, K_{\mathrm{se}}\right)=-\int\left[\langle\beta\rangle\langle\omega\rangle^{*}\langle\alpha\rangle^{*}\right] \rho \mathrm{d} V \\
& C\left(P_{\mathrm{te}}, K_{\mathrm{te}}\right)=-\int\left[\langle\beta\rangle\left\langle\omega^{\prime} \alpha^{\prime}\right\rangle\right] \rho \mathrm{d} V \\
& C\left(K_{\mathrm{se}}, K_{\mathrm{m}}\right)=\int\left[\langle\beta\rangle\langle v\rangle^{*}\langle u\rangle^{*}\right] \cos \phi \frac{\partial([\langle u\rangle] / \cos \phi)}{a \partial \phi} \rho d \mathrm{~V} \\
& +\int\left[\langle\beta\rangle\langle v\rangle^{* 2}\right] \frac{\partial[\langle v\rangle]}{a \partial \phi} \rho \mathrm{d} V \\
& +\int\left[\langle\beta\rangle\langle w\rangle^{*}\langle u\rangle^{*}\right] \frac{\partial[\langle u\rangle]}{\partial p} \rho \mathrm{d} V \\
& +\int\left[\langle\beta\rangle\langle w\rangle^{*}\langle v\rangle^{*}\right] \frac{\partial[\langle v\rangle]}{\partial p} \rho \mathrm{d} V \\
& -\int[\langle v\rangle]\left(\left[\langle\beta\rangle\langle u\rangle^{* 2}\right]\right) \frac{\tan \phi}{a} \rho \mathrm{d} V \\
& C\left(K_{\mathrm{te}}, K_{\mathrm{m}}\right)=\int\left[\langle\beta\rangle\left\langle v^{\prime} u^{\prime}\right\rangle\right] \cos \phi \frac{\partial([\langle u\rangle] / \cos \phi)}{a \partial \phi} \rho \mathrm{d} V \\
& +\int\left[\langle\beta\rangle\left\langle v^{\prime 2}\right\rangle\right] \frac{\partial[\langle v\rangle]}{a \partial \phi} \rho \mathrm{d} V \\
& +\int\left[\langle\beta\rangle\left\langle w^{\prime} u^{\prime}\right\rangle\right] \frac{\partial[\langle u\rangle]}{\partial p} \rho \mathrm{d} V \\
& +\int\left[\langle\beta\rangle\left\langle w^{\prime} v^{\prime}\right\rangle\right] \frac{\partial[\langle v\rangle]}{\partial p} \rho \mathrm{d} V \\
& -\int[\langle v\rangle]\left(\left[\langle\beta\rangle\left\langle u^{\prime 2}\right\rangle\right]\right) \frac{\tan \phi}{a} \rho \mathrm{d} V \\
& C\left(P_{\mathrm{m}}, K_{\mathrm{m}}\right)=-\int[\langle\beta\rangle][\langle\omega\rangle]^{\prime \prime}[\langle\alpha\rangle]^{\prime \prime} \rho \mathrm{d} V .
\end{aligned}
$$

New conversion terms due to the transient and stationary eddy decomposition are:

$$
\begin{gathered}
C\left(P_{\mathrm{te}}, P_{\mathrm{se}}\right)=-c_{\mathrm{p}} \int \gamma\left[\langle\beta\rangle\left\langle T^{\prime} v^{\prime}\right\rangle^{*} \frac{\partial\langle T\rangle^{*}}{a \partial \phi}\right] \rho \mathrm{d} V \\
-c_{\mathrm{p}} \int p^{-k}\left[\langle\beta\rangle\left\langle T^{\prime} \omega^{\prime}\right\rangle^{*} \frac{\gamma p^{\mathrm{k}}\langle T\rangle^{*}}{p}\right] \rho \mathrm{d} V
\end{gathered}
$$




$$
\begin{aligned}
C\left(K_{\mathrm{se}},\right. & \left.K_{\mathrm{te}}\right)=\int \frac{\tan \phi}{a}\left[\langle\beta\rangle\left(\left\langle u^{\prime 2}\right\rangle\langle v\rangle^{*}-\left\langle v^{\prime} u^{\prime}\right\rangle\langle u\rangle^{*}\right)\right] \rho \mathrm{d} V(\mathrm{~A} 15) \\
- & \frac{1}{a} \int\left[\langle\beta\rangle\left(\left\langle u^{\prime} v^{\prime}\right\rangle \frac{\langle u\rangle^{*}}{\partial \phi}+\left\langle v^{\prime 2}\right\rangle \frac{\langle v\rangle^{*}}{\partial \phi}\right)\right] \rho \mathrm{d} V \\
- & {\left[\langle\beta\rangle\left(\left\langle u^{\prime} \omega^{\prime}\right\rangle \frac{\langle u\rangle^{*}}{\partial p}+\left\langle v^{\prime} \omega^{\prime}\right\rangle \frac{\langle v\rangle^{*}}{\partial p}\right)\right] \rho \mathrm{d} V }
\end{aligned}
$$

We do not compute the generation and dissipation rates explicitly, but rather estimate them as residuals. However, we present these expressions here for completeness:

$$
\begin{aligned}
G_{\mathrm{m}} & =\int \gamma[\langle\beta\rangle][\langle T\rangle]^{\prime \prime}[\langle Q\rangle]^{\prime \prime} \rho \mathrm{d} V \\
D_{\mathrm{m}} & =\int[\langle\beta\rangle]\left([\langle u\rangle]\left[\left\langle F_{x}\right\rangle\right]+[\langle v\rangle]\left[\left\langle F_{y}\right\rangle\right]\right) \rho \mathrm{d} V \\
G_{\mathrm{se}} & =\int \gamma\left[\langle\beta\rangle\langle T\rangle^{*}\langle Q\rangle^{*}\right] \rho \mathrm{d} V \\
G_{\mathrm{te}} & =\int \gamma\left[\langle\beta\rangle\left\langle T^{\prime} Q^{\prime}\right\rangle\right] \rho \mathrm{d} V \\
D_{\mathrm{se}} & =\int\left[\langle\beta\rangle\left(\langle u\rangle^{*}\left\langle F_{x}\right\rangle^{*}+\langle v\rangle^{*}\left\langle F_{y}\right\rangle^{*}\right)\right] \rho \mathrm{d} V \\
D_{\mathrm{te}} & =\int\left[\langle\beta\rangle\left(\left\langle u^{\prime} F_{x}^{\prime}\right\rangle+\left\langle v^{\prime} F_{y}^{\prime}\right\rangle\right)\right] \rho \mathrm{d} V .
\end{aligned}
$$

When splitting the atmosphere at an isobaric level, boundary fluxes must be computed for each reservoir. These also include the pressure-work terms. For consistency with our $\omega \alpha$ formulation (see Peixoto and Oort, 1974 for details), the pressure-work terms contribute to the kinetic energy fluxes (last terms in Eqs. A25, A26 and A27). The corresponding expressions are:

$$
\begin{aligned}
B\left(P_{\mathrm{m}}\right) & =c_{\mathrm{p}} \int \gamma\left[\left\langle\omega^{\prime} T^{\prime}\right\rangle+\langle\omega\rangle^{*}\langle T\rangle^{*}\right][\langle T\rangle]^{\prime \prime} \mathrm{d} A / g \\
& +\frac{c_{\mathrm{p}}}{2} \int \gamma[\langle\omega\rangle][\langle T\rangle]^{\prime 2} \mathrm{~d} A / g \\
B\left(P_{\mathrm{se}}\right) & =\frac{c_{\mathrm{p}}}{2} \int \gamma\left[\left\langle\omega\langle T\rangle^{* 2}\right\rangle\right] \mathrm{d} A / g \\
& +c_{\mathrm{p}} \int \gamma\left[\left\langle w^{\prime} T^{\prime}\right\rangle^{*}\langle T\rangle^{*}\right] \mathrm{d} A / g \\
B\left(P_{\mathrm{te}}\right) & =\frac{c_{\mathrm{p}}}{2} \int \gamma\left[\left\langle\omega T^{\prime 2}\right\rangle\right] \mathrm{d} A / g
\end{aligned}
$$

$$
\begin{aligned}
B\left(K_{\mathrm{se}}\right) & =\frac{1}{2} \int\left[\left\langle\omega\left(\langle u\rangle^{* 2}+\langle v\rangle^{* 2}\right)\right\rangle\right] \mathrm{d} A / g \\
& +\int\left[\left\langle\omega^{\prime} u^{\prime}\right\rangle^{*}\langle u\rangle^{*}+\left\langle\omega^{\prime} v^{\prime}\right\rangle^{*}\langle v\rangle^{*}\right] \mathrm{d} A / g \\
& +\int g\left[\langle\omega\rangle^{*}\langle z\rangle^{*}\right] \mathrm{d} A / g \\
B\left(K_{\mathrm{te}}\right) & =\frac{1}{2} \int\left[\left\langle\omega\left(u^{\prime 2}+v^{\prime 2}\right)\right\rangle\right] \mathrm{d} A / g \\
& +\int g\left[\left\langle\omega^{\prime} z^{\prime}\right\rangle\right] \mathrm{d} A / g \\
B\left(K_{\mathrm{m}}\right) & =\frac{1}{2} \int[\langle\omega\rangle]\left([\langle u\rangle]^{2}+[\langle v\rangle]^{2}\right) \mathrm{d} A / g \\
& +\int\left[\left\langle\omega^{\prime} u^{\prime}\right\rangle+\langle\omega\rangle^{*}\langle u\rangle^{*}\right][\langle u\rangle] \mathrm{d} A / g \\
& +\int\left[\left\langle\omega^{\prime} v^{\prime}\right\rangle+\langle\omega\rangle^{*}\langle v\rangle^{*}\right][\langle v\rangle] \mathrm{d} A / g \\
& +\int g[\langle\omega\rangle][\langle z\rangle]^{\prime \prime} \mathrm{d} A / g
\end{aligned}
$$

Acknowledgements. This work was supported by the Max Planck Society and the International Max Planck Research School on Earth System Modelling. Special thanks to Aiko Voigt for helpful comments on the early version of this paper, to the editor, Mathew Huber, and to two anonymous reviewers who contributed to a fruitful discussion.

The service charges for this open access publication have been covered by the Max Planck Society.

Edited by: M. Huber

\section{References}

Boer, G. J.: Diagnostic Equations in Isobaric Coordinates, Mon. Weather Rev., 110, 1801-1820, 1982.

Boer, G. J.: Some Dynamical Consequences of Greenhouse Gas Warming, Atmos.-Ocean, 33, 731-751, 1995.

Boer, G. J. and Lambert, S.: The energy cycle in atmospheric models, Clim. Dynam., 30, 371-390, 2008.

Held, I. M.: Large-Scale Dynamics and Global Warming, B. Am. Meteorol. Soc., 74, 228-240, 1993.

Hernández-Deckers, D. and von Storch, J.-S.: Energetics Responses to Increases in Greenhouse Gas Concentration, J. Climate, 23, 3874-3887, doi:10.1175/2010JCLI3176.1, 2010.

Holton, J. R.: An Introduction to Dynamical Meteorology, Elsevier Inc., USA, 2004.

Li, L., Ingersoll, A. P., Jiang, X., Feldman, D., and Yung, Y. L.: Lorenz energy cycle of the global atmosphere based on reanalysis datasets, Geophys. Res. Lett., 34, L16813, doi:10.1029/2007GL029985, 2007.

Lorenz, E. N.: Available potential Energy and the Maintenance of the General Circulation, Tellus, 7, 157-167, 1955. 
Lucarini, V.: Thermodynamic efficiency and entropy production in the climate system, Phys. Rev. E, 80, 021118, doi:10.1103/PhysRevE.80.021118, 2009.

Lucarini, V., Fraedrich, K., and Lunkeit, F.: Thermodynamics of climate change: generalized sensitivities, Atmos. Chem. Phys., 10, 9729-9737, doi:10.5194/acp-10-9729-2010, 2010.

Marquet, P.: Diagnostic of change in energetics for a $2 \times \mathrm{CO}_{2}$, EGU, Vienna, personal communication, 2006.

Marsland, S., Haak, H., Jungclaus, J., Latif, M., and Roske, F.: The Max-Planck-Institute global ocean/sea ice model with orthogonal curvilinear coordinates, Ocean Modell., 5, 91-127, 2003.

Meehl, G., Stocker, T., Collins, W., Friedlingstein, P., Gaye, A., Gregory, J., Kitoh, A., Knutti, R., Murphy, J., Noda, A., Raper, S., Watterson, I., Weaver, A., and Zhao, Z.-C.: Global Climate Projections, in: Climate Change 2007: The Physical Science Basis, Contribution of Working Group I to the Fourth Assesment Report of the Intergovernmental Panel on Climate Change, edited by: Solomon, S., Qin, D., Manning, M., Chen, Z., Marquis, M., Averyt, K., Tignor, M., and Miller, H., Cambridge University Press, Cambridge, UK and New York, NY, USA, 747845, 2007.

O'Gorman, P. A.: Understanding the varied response of the extratropical storm tracks to climate change, P. Natl. Acad. Sci. USA, 107(45), 19176-19180, doi:10.1073/pnas.1011547107, 2010.

O'Gorman, P. A. and Schneider, T.: Energy of Midlatitude Transient Eddies in Idealized Simulations of Changed Climates, J. Climate, 21, 5797-5806, doi:10.1175/2008JCLI2099.1, 2008.

Oort, A. H. and Peixoto, J. P.: The Annual Cycle of the Energetics of the Atmosphere on a Planetary Scale, J. Geophys. Res., 79, 2705-2719, 1974.
Peixoto, J. P. and Oort, A. H.: The Annual Distribution of Atmospheric Energy on a Planetary Scale, J. Geophys. Res., 79, 21492159, 1974.

Peixoto, J. P. and Oort, A. H.: Physics of Climate, American Institute of Physics, New York, USA, 1992.

Roeckner, E.: IPCC-AR4 MPI-ECHAM5_T63L31 MPIOM_GR1.5L40 1\%/year CO2 increase experiment to doubling run no. 1: atmosphere 6 HOUR values MPImet/MaD Germany, http://cera-www.dkrz.de/WDCC/ui/Compact.jsp?acronym= EH5-T63L31_OM_1CO2_1_6H (last access: 20 June 2011), 2004.

Roeckner, E., Bauml, G., Bonavontura, L., Brokopf, R., Esch, M., Giorgetta, M., Hagemann, S., Kirchner, I., Kornblueh, L., Manzini, E., Rhodin, A., Schlese, U., Schulzweida, U., and Tompkins, A.: The atmospheric general circulation model ECHAM5, Part I: Model description, Tech. Rep. 349, MaxPlanck-Institute for Meteorology, 2003.

Roeckner, E., Lautenschlager, M., and Esch, M.: IPCCAR4 MPI-ECHAM5_T63L31 MPI-OM_GR1.5L40 PIcntrl (pre-industrial control experiment): atmosphere 6 HOUR values MPImet/MaD Germany, doi:10.1594/WDCC/EH5T63L31_OM-GR1.5L40_CTL_6H, 2006.

Romanski, J.: Investigating the Role of Individual Diabatic Heating Components in Global Atmospheric Circulation and Climate Sensitivity: An Energetics Approach, Ph.d. thesis, Columbia University, New York, NY, 2009.

von Storch, J.-S.: Towards climate prediction: Interannual potential predictability due to an increase in $\mathrm{CO}_{2}$ concentration as diagnosed from an ensemble of AO-GCM integrations, J. Climate, 21, 4607-4628, 2008. 